\title{
TOPOLOGICAL RIGIDITY FOR FJ BY THE INFINITE CYCLIC GROUP
}

\author{
KUN WANG
}

\begin{abstract}
We call a group FJ if it satisfies the $K$ - and $L$-theoretic Farrell-Jones conjecture with coefficients in $\mathbb{Z}$. We show that if $G$ is FJ, then the simple Borel conjecture (in dimensions $\geq 5$ ) holds for every group of the form $G \rtimes \mathbb{Z}$. If in addition $W h(G \times \mathbb{Z})=0$, which is true for all known torsion free FJ groups, then the bordism Borel conjecture (in dimensions $n \geq 5$ ) holds for $G \rtimes \mathbb{Z}$. One of the key ingredients in proving these rigidity results is another main result, which says that if a torsion free group $G$ satisfies the $L$-theoretic Farrell-Jones conjecture with coefficients in $\mathbb{Z}$, then any semi-direct product $G \rtimes \mathbb{Z}$ also satisfies the $L$-theoretic Farrell-Jones conjecture with coefficients in $\mathbb{Z}$. Our result is indeed more general and implies the $L$-theoretic Farrell-Jones conjecture with coefficients in additive categories is closed under extensions of torsion free groups. This enables us to extend the class of groups which satisfy the Novikov conjecture.
\end{abstract}

\section{INTRODUCTION}

In the area of manifold topology, one of the most intriguing unsolved problem is the Borel conjecture on topological rigidity of closed aspherical manifolds. Recall that a manifold is called aspherical if its universal cover is contractible.

The Borel Conjecture. Every closed aspherical manifold is topologically rigid. That is, every homotopy equivalence between any two closed aspherical manifolds is homotopic to a homeomorphism.

Note that every homeomorphism between two closed manifolds is simple, i.e. its Whitehead torsion vanishes. This follows from a theorem of Chapman [10] which says that every homeomorphism between two finite $C W$-complexes is simple. Therefore, it is reasonable to study the simple version of the Borel conjecture, which is the converse (up to homotopy) to Chapman's topological invariance theorem of Whitehead torsion for closed aspherical manifolds:

The Simple Borel Conjecture. Every closed aspherical manifold is simply topologically rigid. That is, every simple homotopy equivalence between any two closed aspherical manifolds is homotopic to a homeomorphism.

Clearly, the simple Borel conjecture is simpler than the Borel conjecture. The passage from the simple Borel conjecture to the Borel conjecture is the famous conjecture which states that the Whitehead group of any torsion free group vanishes (note that the fundamental group of a closed aspherical manifold is torsion free). 
Another type of rigidity question one can ask for aspherical manifold is the following bordism type rigidity:

The Bordism Borel Conjecture: Every closed aspherical manifold is bordismly topologically rigid. That is, every homotopy equivalence $f: N \longrightarrow M$ from another closed manifold $N$ to $M$ is $h$-cobordant to the identity $1: M \longrightarrow M$.

Recall that a cobordism $\left(W ; M_{1}, M_{2}\right)$ is called an $h$-corbordim if the inclusions $M_{1} \hookrightarrow W, M_{2} \hookrightarrow$ $W$ are homotopy equivalence. Two homotopy equivalences $f_{i}: M_{i} \longrightarrow X, i=1,2$ are called $h$-cobordant if there is an $h$-cobordism $\left(W ; M_{1}, M_{2}\right)$ and a homotopy equivalence $\left(F ; f_{1}, f_{2}\right)$ : $\left(W ; M_{1}, M_{2}\right) \longrightarrow(X \times[0,1] ; X \times 0, X \times 1)$. Note that, if we require the map $f$ to be simple and $h$-cobordant to be $s$-cobordant in the bordism Borel conjecture, we then get the $s$-cobordism version of the Borel conjecture, which by the $s$-cobordism theorem, is just the simple Borel conjecture in dimensions $\geq 5$.

From the viewpoint of surgery theory, the simple version and the bordism version of the Borel conjecture are very natural, since they have direct connection to the surgery long exact sequences. We refer the reader to [24] for a survey on aspherical manifolds and [17] for a discussion about the above three versions of the Borel conjecture.

For convenience, we introduce the following. We say the Borel conjecture, or the simple Borel conjecture, or the bordism Borel conjecture, holds for a group $G$ if either $G$ cannot be realized as the fundamental group of a closed aspherical manifold, or the respective conjecture holds for every closed aspherical manifold with fundamental group isomorphic to $G$. It is an interesting problem to identify which groups can be realized as the fundamental group of a closed aspherical manifold. Conjecturally, a group has this property if and only if the group is a finitely presented Poincaré duality group. This conjecture is usually referred to as Wall's conjecture (though Wall [29] did not include the finitely presented condition, which would otherwise make the conjecture false). See [12] for a survey.

In recent years, there has been significant progress on the proof of the Borel conjecture for a large class of groups, due to the solutions of the Farrell-Jones conjecture (FJC for short) for these groups. See the works by many authors [2], [5], [6], [7], 31], 30], 22], [18], [16], 15], [14, [28]. Roughly speaking, the conjecture says that the algebraic $K$ - and $L$-groups $K_{n}(\mathbb{Z}[G]), L_{n}(\mathbb{Z}[G]), n \in \mathbb{Z}$ of the integral group ring $\mathbb{Z}[G]$ of a group $G$ is determined by those of its virtually cyclic subgroups and the group homology of $G$. The conjecture was first formulated in [13] by Farrell and Jones with coefficients in $\mathbb{Z}$. Then in [11], Davis and Lück gave a general framework for the formulations of various isomorphism conjectures in $K$-and $L$-theories. In these formulations, the coefficients are untwisted rings. Later on, the conjecture was extended by Bartels and Reich [8] to allow for coefficients in any additive category $\mathcal{A}$ with a right $G$-action. The precise formulation of the conjecture will be given in Section 2.1, 
The significance of the Farrell-Jones conjecture not only lies in the fact that it provides a tool for the computations of algebraic $K$ - and $L$-theories of groups rings, but also implies many important conjectures in geometry, topology and algebra. In particular, if both the $K$ - and $L$-theoretic FJC with coefficients in $\mathbb{Z}$ hold for a group $G$, then the Borel conjecture holds for $G$ in dimensions greater than or equal to 5 . If the $L$-theoretic FJC with coefficients in $\mathbb{Z}$ holds for $G$, then the Novikov conjecture on homotopy invariance of higher signatures holds for $G$. See [25] for a survey on the FJC and its applications.

We call a group $F J$ if it satisfies the $K$ - and $L$-theoretic FJC with coefficients in $\mathbb{Z}$. We denote the class of all FJ groups by $\mathcal{F} \mathcal{J}$. In general, in application of the FJC to the Borel conjecture, one needs to show $G \in \mathcal{F} \mathcal{J}$ if one wants to prove the Borel conjecture for $G$ in dimensions $\geq 5$. Interestingly, one of our main result shows the following:

Theorem A. If $G \in \mathcal{F} \mathcal{J}$, then the simple Borel conjecture holds for every semi-direct product $G \rtimes \mathbb{Z}$ in dimensions $\geq 5$. If in addition $W h(G \times \mathbb{Z})=0$, then the bordism Borel conjecture holds for every semi-direct product $G \rtimes \mathbb{Z}$ in dimensions $\geq 5$.

An immediate corollary of Theorem A is the following:

Corollary 1.1. Let $M$ be a closed aspherical manifold which fibers over the unit circle with fiber $N$. If $\pi_{1}(N) \in \mathcal{F} \mathcal{J}$ and $\operatorname{dim}(M) \geq 5$, then the simple Borel conjecture holds for $M$. If in addition $W h\left(\pi_{1}(N) \times \mathbb{Z}\right)=0$, then the bordism Borel conjecture holds for $M$.

Following [5], we define a class of groups $\mathcal{B}$ in the following way, except we add more groups to the list.

Definition 1.2. Let $\mathcal{B}$ be the smallest class of groups so that:

(1) $\mathcal{B}$ contains all groups from the following classes of groups: CAT(0) groups, Gromov hyperbolic groups, lattices in virtually connected Lie groups, virtually solvable groups, fundamental groups of graphs of abelian groups, $S$-arithmetic groups.

(2) $\mathcal{B}$ is closed under taking subgroups, finite direct products of groups, free products of groups, direct colimits (with not necessary injective structure maps) of groups.

(3) For any group homomorphism $\phi: G \rightarrow H$. If $H \in \mathcal{B}$ and $\phi^{-1}(V) \in \mathcal{B}$ for every virtually cyclic subgroup $V \subseteq H$, then $G \in \mathcal{B}$.

Corollary 1.3. If $G \in \mathcal{B}$, then the simple Borel conjecture and the bordism Borel conjecture holds for every semi-direct product $G \rtimes \mathbb{Z}$ in dimensions $\geq 5$.

Proof. Note that we can assume $G$ is torsion free, otherwise $G$ is not the fundamental group of any closed aspherical manifold. Now we have $\mathcal{B} \subseteq \mathcal{F} \mathcal{J}$ since the groups in part (1) of Definition 1.2 and the groups obtained from these groups by the operations in parts (2) and (3) satisfy the $K$ and $L$-theoretic FJC with coefficients in $\mathbb{Z}$ (with coefficients in any additive category indeed), see 
[5], [6], [31, [30, 22, [22, [18, [16, [15, [28]. Therefore, Corollary 1.3 follows from Theorem A and the fact that $W h(G \times \mathbb{Z})=0$ if $G$ is torsion free and $G \in \mathcal{B}$. This is because $G \times \mathbb{Z} \in \mathcal{B} \subseteq \mathcal{F} \mathcal{J}$ and Whitehead groups of torsion free FJ groups vanish.

One of the main ingredients in proving Theorem A is the following result, which is of course of independent interest:

Theorem B. Let $G$ be a torsion free group and $G \rtimes \mathbb{Z}$ be any semi-direct product of $G$ with $\mathbb{Z}$.

(1) Suppose the L-theoretic FJC with coefficients in $\mathbb{Z}$ holds for $G$, then it also holds for $G \rtimes \mathbb{Z}$.

(2) Suppose the $K$-theoretic FJC with coefficients in $\mathbb{Z}$ holds for $G$, then there are obstruction

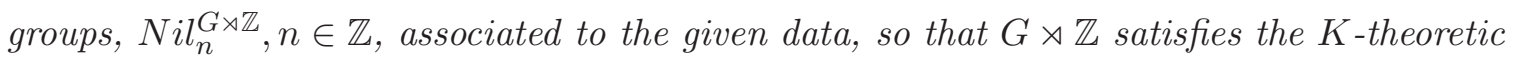

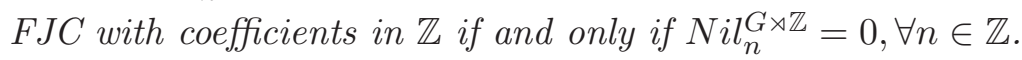

Remark 1.4. As one sees, we are not able to prove the same result as in part (1) of Theorem B for the $K$-theoretic FJC with coefficients in $\mathbb{Z}$. Therefore, some additional argument is needed in order to deduce Theorem A from Theorem B. This is achieved by proving some general result on $L$-groups. See Lemma 4.1 .

Remark 1.5. Our result is indeed more general. It still holds if we replace the coefficient ring $\mathbb{Z}$ by any right $G$-additive category $\mathcal{A}$ with involution in part (1) and replace the coefficient ring $\mathbb{Z}$ by any associative ring with unit which is regular in part (2) (a ring is called regular if it is left Noetherian and every finitely generated left $R$-module has a projective resolution of finite type). For example, let $\mathcal{A}$ be a right $G \rtimes \mathbb{Z}$-additive category with involution, view $\mathcal{A}$ as a right $G$-additive category with involution in the natural way. Then if the $L$-theoretic FJC with coefficient in $\mathcal{A}$ holds for $G$, the $L$-theoretic FJC with coefficient in $\mathcal{A}$ also holds for $G \rtimes \mathbb{Z}$.

Remark 1.6. A key step in the proof of FJC for some classes of groups, including the class of Baumslag-Solitar groups [16], [15] and the class of solvable groups [30, is to prove the conjecture for a group of the form $G \rtimes_{\alpha} \mathbb{Z}$, where $G$ is a torsion free abelian group and $\alpha$ is some special action of $\mathbb{Z}$ on $G$. Complicated geometric arguments are used in these works in order to prove the conjecture for $G \rtimes_{\alpha} \mathbb{Z}$. Therefore, a general result as in part (1) of Theorem B is very useful.

In the literature, the term FJC may refer to different versions of the conjecture and they have different inheritance properties. For convenience, we introduce the following. Let $G$ be a group and $\mathcal{A}$ be a right $G$-additive category. We say a group $G$ satisfies FJC $_{\mathcal{A}}$ if $G$ satisfies FJC with coefficient in $\mathcal{A}$. We say $G$ satisfies $\mathrm{FJC}_{A d}$ if it satisfies $\mathrm{FJC}_{\mathcal{A}}$ for every additive category $\mathcal{A}$ with a right $G$-action. When $\mathcal{A}$ is the category of finitely generated left free $R$-modules for some associative ring $R$ with trivial group action, we then denote $\mathrm{FJC}_{\mathcal{A}}$ by $\mathrm{FJC}_{R}$.

The version $\mathrm{FJC}_{A d}$ has some nice inheritance properties. For example, $\mathrm{FJC}_{A d}$ is closed under the operations in parts (2) and (3) of Definition 1.2, see [2, Section 2.3] for a summary. However, these inheritance properties are unknown for $\mathrm{FJC}_{\mathcal{A}}$; except it was proved in [3, Theorem 0.8] that 
$\mathrm{FJC}_{\mathcal{A}}$ is closed under taking direct colimit of groups with injective structure maps. Theorem $\mathrm{B}$ is probably the second inheritance property for the $L$-theoretic $\mathrm{FJC}_{\mathcal{A}}$.

Theorem $\mathrm{B}$ together with some nice inheritance properties of $\mathrm{FJC}_{A d}$ implies the following:

Corollary 1.7. Let $1 \longrightarrow K \longrightarrow G \longrightarrow Q \longrightarrow 1$ be an extension of groups. Suppose both $K$ and $Q$ are torsion free and satisfy the $L$-theoretic $F J C_{A d}$, then $G$ also satisfies the $L$-theoretic $F J C_{A d}$.

Proof. It is well-known that an infinite virtually cyclic group either admits a surjection onto the infinite cyclic group with finite kernel or admits a surjection onto the infinite Dihedral group with finite kernel. Therefore a torsion free virtually cyclic group is either trivial or infinite cyclic. Hence, by the fact that $\mathrm{FJC}_{A d}$ is closed under operation (3) in Definition [1.2, see [2, Theorem 2.7][19, Theorem $\mathrm{A}]$, it suffices to show that for any infinite cyclic subgroup of $Q$, its inverse image in $G$ satisfies the $L$-theoretic $\mathrm{FJC}_{A d}$. Note that its inverse image in $G$ is isomorphic to a group of the form $K \rtimes_{\alpha} \mathbb{Z}$. Let $\mathcal{A}$ be any additive $K \rtimes_{\alpha} \mathbb{Z}$ category. By assumption, $K$ is torsion free and satisfies the $L$-theoretic $\mathrm{FJC}_{\mathcal{A}}$, so by Theorem B and Remark 1.5, $K \rtimes_{\alpha} \mathbb{Z}$ also satisfies the $L$-theoretic $\mathrm{FJC}_{\mathcal{A}}$. Since $\mathcal{A}$ can be arbitrary, we see that $K \rtimes_{\alpha} \mathbb{Z}$ satisfies $\mathrm{FJC}_{A d}$. This completes the proof.

Let $\mathcal{C}$ be a class of groups. A group $G$ is called poly-C if it has a filtration by subgroups

$$
1=G_{0} \subseteq G_{1} \subseteq G_{2} \subseteq \cdots \subseteq G_{n}=G
$$

so that each $G_{i-1}$ is normal in $G_{i}, i=1,2, \cdots, n$ and each $G_{i} / G_{i-1}$ is in $\mathcal{C}$.

Definition 1.8. Let $\mathcal{B}$ be as in Definition 1.2, Define $\mathcal{D} \subseteq \mathcal{B}$ to be the class of groups in $\mathcal{B}$ which are torsion free.

Corollary 1.9. The class of poly-D-groups satisfies the L-theoretic $F J C_{A d}$. Therefore, they also satisfy the Novikov conjecture.

Proof. By repeatedly applying Corollary 1.7, we see that the $L$-theoretic $\mathrm{FJC}_{A d}$ holds for poly-D groups.

Remark 1.10. The best result so far for the Novikov conjecture is due to Guoliang Yu, who proved in [32] the coarse Baum-Connes conjecture for groups which admit a uniform embedding into Hilbert space. This is a huge class of groups. However, there are groups, constructed as direct colimits of Gromov hyperbolic groups, which do not admit a uniform embedding into Hilbert space, see for example [20]. Note that these groups lie in the class $\mathcal{B}$. Also it is still an open question that whether CAT(0)-groups and their extensions admit a uniform embedding into Hilbert space. Therefore, poly-D-groups contain new examples of groups that satisfy the Novikov conjecture.

The paper is organized as follows. In Section 2, we briefly recall the formulation of the FarrellJones conjecture and the controlled algebra approach to the conjecture. We use this approach 
to prove Theorem B. We also prove some lemmas on the equivariantly continuously controlled condition. These lemmas are important in proving the main theorem (Theorem 3.6) of Section 3 , which is a key step in proving Theorem B. We prove our main theorems in the final section, in which we first prove an important lemma (Lemma 4.1) for $L$-groups. This lemma together with Theorem B enable us to prove Theorem A.

Acknowledgement. The author would like to thank Professor Bruce Hughes for his support and helpful comments about the paper. The author would also like to thank his thesis advisor Professor Jean-François Lafont for his enormous and continuous support throughout the years. Part of the project was initiated when the author was a graduate student at the Ohio State University under the guidance of Jean. The author also thanks Professor Guoliang Yu for his support and encouragement. The author learned a lot through numerous discussions with Guoliang. The author also thanks Shanghai Center for Mathematical Science for its hospitality during the author's visit in Summer 2015. Part of the project was done during this visit.

\section{The FARrell-Jones CONJECTURe AND CONTROLled AlgeBrA}

In the first half of the section, we briefly recall the formulation of the FJC and the controlled algebra approach to the conjecture. More details can be found in [13, [11, [4, 8]. This approach will be important to our treatment later. In the second half of the section, we prove some lemmas on the equivariantly continuously controlled condition (see Definition 2.2.) that will be important in later sections.

2.1. The Farrell-Jones conjecture. Let $G$ be a group and $\mathcal{A}$ be an additive category with a right $G$-action $\alpha$ (we always assume $\mathcal{A}$ comes with an involution, which is compatible with the right $G$-action, when we talk about $L$-theory). One can form the "twisted group additive category" $\mathcal{A}_{\alpha}[G]$ [8, Definition 2.1] (this category is denoted by $\mathcal{A} *_{G} p t$ in [8], but it is more enlightening to denote this category by $\mathcal{A}_{\alpha}[G]$ for our purpose). Its definition is recalled in Definition 3.1 and it will be important to our treatment later. When $\mathcal{A}$ is the additive category of finitely generated left free $R$-modules with the trivial $G$-action, $\mathcal{A}[G]$ is equivalent to the additive category of finitely generated left free $R[G]$-modules. Now let $H_{*}^{G}\left(-; \mathbf{K}_{\mathcal{A}}\right)$ and $H_{*}^{G}\left(-; \mathbf{L}_{\mathcal{A}}^{<-\infty>)}\right.$ be the two $G$-equivariant homology theories constructed by Bartels and Reich in [8], using the method of Davis and Lück [11]. These two equivariant homology theories have the property that for any subgroup $H<G, H_{n}^{G}\left(G / H ; \mathbf{K}_{\mathcal{A}}\right)=K_{n}\left(\mathcal{A}_{\alpha}[H]\right), H_{n}^{G}\left(G / H ; \mathbf{L}_{\mathcal{A}}^{<-\infty>}\right)=L_{n}^{<-\infty>}\left(\mathcal{A}_{\alpha}[H]\right), \forall n \in \mathbb{Z}$, where the decoration $-\infty$ on the $L$-groups means we are dealing with Ranicki's ultimate $L$-groups. In particular, $H_{n}^{G}\left(p t ; \mathbf{K}_{\mathcal{A}}\right)=K_{n}\left(\mathcal{A}_{\alpha}[G]\right)$ and $H_{n}^{G}\left(p t ; \mathbf{L}_{\mathcal{A}}^{<-\infty>}\right)=L_{n}^{<-\infty>}\left(\mathcal{A}_{\alpha}[G]\right)$.

We also need the notion of classifying space of a group relative to a family of its subgroups. For $\mathcal{F}$, a family of subgroups of $G$ which is closed under taking subgroups and conjugations, denote by $E_{\mathcal{F}} G$ a model for the classifying space of $G$ relative to the family $\mathcal{F}$. It is a $G$-CW complex and 
is characterized, up to $G$-equivariant homotopy equivalence, by the properties that every isotropy group of the action lies in the family $\mathcal{F}$, and the fixed point set of $H$ is contractible if $H \in \mathcal{F}$ and empty if $H \notin \mathcal{F}$. Examples of classifying spaces are $E G, E_{\mathcal{F I N}} G$, and $E_{\mathcal{V} C} G$, corresponding to the families of trivial, finite, and virtually cyclic subgroups of $G$ respectively.

The Farrell-Jones Conjecture. Let $G$ be a group and $\mathcal{A}$ be an additive category with a right $G$ action. We say the group $G$ satisfies the $K$-theoretic FJC with coefficient in $\mathcal{A}$ if the $K$-theoretic assembly map

$$
A_{K}^{\mathcal{A}}: H_{n}^{G}\left(E_{\mathcal{V C}} G ; \mathbf{K}_{\mathcal{A}}\right) \rightarrow H_{n}^{G}\left(p t ; \mathbf{K}_{\mathcal{A}}\right)=K_{n}\left(\mathcal{A}_{\alpha}[G]\right)
$$

which is induced by the obvious map $E_{\mathcal{V C}} G \rightarrow p t$, is an isomorphism for all $n \in \mathbb{Z}$. We say the group $G$ satisfies the $L$-theoretic FJC with coefficient in $\mathcal{A}$ if the corresponding $L$-theoretic assembly map

$$
A_{L}^{\mathcal{A}}: H_{n}^{G}\left(E_{\mathcal{V C}} G ; \mathbf{L}_{\mathcal{A}}^{<-\infty>}\right) \rightarrow H_{n}^{G}\left(p t ; \mathbf{L}_{\mathcal{A}}^{<-\infty>}\right)=L_{n}^{<-\infty>}\left(\mathcal{A}_{\alpha}[G]\right)
$$

is an isomorphism for all $n \in \mathbb{Z}$.

2.2. Controlled algebra approach to the FJC. Let $\mathcal{A}$ be a (small) additive category with a right $G$-action and $X$ be a left $G$-space. The additive category $\mathcal{C}(X ; \mathcal{A})$ of geometric modules over $X$ with coefficient in $\mathcal{A}$ is defined as follows: objects are functions $A: X \rightarrow O b(\mathcal{A})$ with locally finite support, i.e. $\operatorname{supp} A=\left\{x \in X \mid A_{x} \neq 0\right\}$ is a locally finite subset of $X$, meaning every point in $X$ has an open neighborhood whose intersection with $\operatorname{supp} A$ is finite. An object will usually be denoted by $A=\left(A_{x}\right)_{x \in X}$. A morphism $\phi: A \rightarrow B$ is a matrix of morphisms $\left(\phi_{y, x}: A_{x} \rightarrow B_{y}\right)_{(x, y) \in X \times X}$ such that there are only finitely many nonzero entries in each row and each column. Compositions of morphisms are given by matrix multiplications. More precisely

$$
\left(\psi_{z, y}: B_{y} \rightarrow C_{z}\right) \circ\left(\phi_{y, x}: A_{x} \rightarrow B_{y}\right)=\left(\sum_{y \in X} \psi_{z, y} \circ \phi_{y, x}: A_{x} \rightarrow C_{z}\right)
$$

Note that the sum on the right hand side is finite. There is a right $G$-action on $\mathcal{C}(X ; \mathcal{A})$, which is given by

$$
\left(g^{*} A\right)_{x}=g^{*}\left(A_{g x}\right),\left(g^{*} \phi\right)_{y, x}=g^{*}\left(\phi_{g y, g x}\right)
$$

and the fixed category is denoted by $\mathcal{C}^{G}(X ; \mathcal{A})$.

For any object $A$ and morphism $\phi$ in $\mathcal{C}(X ; \mathcal{A})$, their supports are the following sets:

$$
\operatorname{supp} A=\left\{x \in X \mid A_{x} \neq 0\right\}, \quad \operatorname{supp} \phi=\left\{(x, y) \in X \times X \mid \phi_{y, x} \neq 0\right\}
$$

In order to get interesting subcategories of $\mathcal{C}(X ; \mathcal{A})$, one can prescribe certain support conditions on objects and morphisms. The convenient language for this purpose is the notion of coarse structures on spaces introduced in [21]. We recall the definition here (with a slight modification for our purpose). 
Definition 2.1. A coarse structure $(\mathcal{E}, \mathcal{F})$ on a set $X$ is a collection $\mathcal{E}$ of subsets of $X \times X$, and a collection $\mathcal{F}$ of subsets of $X$ satisfying the following properties:

(1) If $E^{\prime}, E^{\prime \prime} \in \mathcal{E}$, then $E^{\prime} \cup E^{\prime \prime} \subseteq E$ for some $E \in \mathcal{E}$;

(2) If $E^{\prime}, E^{\prime \prime} \in \mathcal{E}$, then $E^{\prime} \circ E^{\prime \prime}=\left\{(x, y) \in X \times X \mid \exists z \in X\right.$ s.t. $(x, z) \in E^{\prime}$ and $\left.(z, y) \in E^{\prime \prime}\right\} \subseteq E$ for some $E \in \mathcal{E}$;

(3) The diagonal $\Delta=\{(x, x) \mid x \in X\}$ is contained in some $E \in \mathcal{E}$;

(4) If $F^{\prime}, F^{\prime \prime} \in \mathcal{F}$, then $F^{\prime} \cup F^{\prime \prime} \subseteq F$ for some $F \in \mathcal{F}$.

If there is a $G$-action on the set $X$, we then require every member in $\mathcal{E}$ and $\mathcal{F}$ to be $G$-invariant, where $G$ acts on $X \times X$ diagonally. If $p: Y \rightarrow X$ is a $G$-equivariant map, then the pullback $\left((p \times p)^{-1} \mathcal{E}, p^{-1} \mathcal{F}\right)$ is a coarse structure on $Y$.

Now if $(\mathcal{E}, \mathcal{F})$ is a coarse structure on a $G$-space $X$, one can define a subcategory $\mathcal{C}(X, \mathcal{E}, \mathcal{F} ; \mathcal{A})$ of $\mathcal{C}(X ; \mathcal{A})$, with object and morphism supports contained in members of $\mathcal{F}$ and $\mathcal{E}$ respectively (in addition to the general finiteness conditions on them). $G$ acts on this additive subcategory and the fixed subcategory is denoted by $\mathcal{C}^{G}(X, \mathcal{E}, \mathcal{F} ; \mathcal{A})$. The pair $(\mathcal{E}, \mathcal{F})$ are usually referred to as control conditions on morphisms and objects.

One of the control condition on morphisms, which is used to construct a model for the assembly maps in the FJC, is the equivariantly continuously controlled condition introduced in [4. It is a generalization to the equivariant setting of the continuously controlled condition introduced in [1. We recall the definition here.

Definition 2.2. ([4, Definition 2.7]) Let $X$ be a topological space with a left $G$-action by homeomorphisms. Equip $X \times[1, \infty]$ with the diagonal $G$-action, where $G$-acts trivially on $[1, \infty]$. A subset $E \subseteq(X \times[1, \infty))^{2}$ is called equivariantly continuously controlled if the following holds:

(i) For every $x \in X$ and $G_{x}$-invariant open neighborhood $U$ of $(x, \infty)$ in $X \times[1, \infty]$, there exists a $G_{x}$-invariant neighborhood $V \subseteq U$ of $(x, \infty)$ in $X \times[1, \infty]$ such that

$$
\left(U^{c} \times V\right) \cap E=\emptyset
$$

where $U^{c}$ denotes the complement of $U$ in $X \times[1, \infty]$.

(ii) There exists $\alpha>0$, depending on $E$, such that if $(x, s) \times\left(x^{\prime}, s^{\prime}\right) \in E$, then $\left|s-s^{\prime}\right|<\alpha$;

(iii) $E$ is symmetric, i.e. if $(p, q) \in E$, then $(q, p) \in E$;

(iv) $E$ is invariant under the diagonal action of $G$.

The collection of $G$-equivariantly continuously controlled subsets of $(X \times[1, \infty))^{2}$ will be denoted by $\mathcal{E}_{G c c}^{X}$. It satisfies the conditions (1)-(3) in Definition 2.1.

Definition 2.3. ([4, Section 3.2] [6, Section 3.3]) For any left $G$-space $X$ and additive category $\mathcal{A}$ with a right $G$-action, one defines the following categories:

(1) $\mathcal{O}^{G}(X ; \mathcal{A})=\mathcal{C}^{G}\left(G \times X \times[1, \infty),(p \times p)^{-1} \mathcal{E}_{G c c}^{X} \cap(r \times r)^{-1} \mathcal{E}_{G}, q^{-1} \mathcal{F}_{G c} ; \mathcal{A}\right)$, where $p: G \times X \times$ $[1, \infty) \rightarrow X \times[1, \infty), q: G \times X \times[1, \infty) \rightarrow G \times X$ and $r: G \times X \times[1, \infty) \rightarrow G$ are projections, $\mathcal{E}_{G}=$ 
$\left\{E \subseteq G \times G \mid g E=E\right.$ for all $g \in G$ and $\exists$ finite $S \subseteq G$ s.t. for all $\left(g, g^{\prime}\right) \in E$, we have $\left.g^{-1} g^{\prime} \in S\right\}$ and $\mathcal{F}_{G c}$ consists of $G$-cocompact subsets of $G \times X$, i.e. subsets of the form $G \cdot K \subseteq G \times X$, where $K \subseteq G \times X$ is compact;

$(2) \mathcal{T}^{G}(X ; \mathcal{A})$ is the full subcategory of $\mathcal{O}^{G}(X ; \mathcal{A})$ consisting of those objects $A$ with the following property: there exists $C>1$ such that if $A_{(g, x, t)} \neq 0$, then $t<C$;

(3) $\mathcal{D}^{G}(X ; \mathcal{A})$ is the quotient category of $\mathcal{O}^{G}(X ; \mathcal{A})$ by the full subcategory $\mathcal{T}^{G}(X ; \mathcal{A})$ : it has the same objects as $\mathcal{O}^{G}(X ; \mathcal{A})$, and any morphism from $A$ to $B$ in $\mathcal{D}^{G}(X ; \mathcal{A})$ is represented by a morphism $\phi: A \rightarrow B$ in $\mathcal{O}^{G}(X ; \mathcal{A})$, with two morphisms $\phi, \psi: A \rightarrow B$ identified if their difference $\phi-\psi$ factors through an object in $\mathcal{T}^{G}(X ; \mathcal{A})$.

Remark 2.4. In [4], the morphism control condition $\mathcal{E}_{G}$ was not required in the definitions. It was added into the definitions in [6] [5] because it was important for proving the conjecture for hyperbolic and CAT(0)-groups. Although this addition changes the categories, it does not change the theory. Later on, we will see that this is also important for our treatment.

These constructions define functors from the category of $G$-CW complexes to the category of additive categories. The importance of these categories lies in the following facts:

Theorem 2.5. The following results hold:

(i) The sequence

$$
\mathcal{T}^{G}(X ; \mathcal{A}) \rightarrow \mathcal{O}^{G}(X ; \mathcal{A}) \rightarrow \mathcal{D}^{G}(X ; \mathcal{A})
$$

is a Karoubi filtration, hence gives rise to a fibration sequence

$$
\mathbb{K}^{-\infty}\left(\mathcal{T}^{G}(X ; \mathcal{A})\right) \rightarrow \mathbb{K}^{-\infty}\left(\mathcal{O}^{G}(X ; \mathcal{A})\right) \rightarrow \mathbb{K}^{-\infty}\left(\mathcal{D}^{G}(X ; \mathcal{A})\right)
$$

of spectra after applying the non-connective $K$-theory, and therefore a long exact sequence on $K$ groups;

(ii) The additive category $\mathcal{O}^{G}(p t ; \mathcal{A})$ has trival $K$-groups;

(iii) The functor $\pi_{*}: \mathcal{T}^{G}(X ; \mathcal{A}) \rightarrow \mathcal{T}^{G}(p t ; \mathcal{A})$ induced by the obvious map $\pi: X \rightarrow\{p t\}$ is an equivalence of categories, hence induces isomorphisms on $K$-groups;

(iv) There is a natural isomorphism between the two functors $H_{*}^{G}\left(-; \boldsymbol{K}_{\mathcal{A}}\right)$ and $K_{*+1}\left(\mathcal{D}^{G}(-; \mathcal{A})\right):=$ $\pi_{*+1}\left(\mathbb{K}^{-\infty}\left(\mathcal{D}^{G}(-; \mathcal{A})\right)\right)$ from the category of $G-C W$ complexes to the category of graded abelian groups. In particular, the map

$$
K_{*+1}\left(\mathcal{D}^{G}\left(E_{\mathcal{V C}} G ; \mathcal{A}\right)\right) \rightarrow K_{*+1}\left(\mathcal{D}^{G}(p t ; \mathcal{A})\right)
$$

is equivalent to the assembly map 2.1.

For information about Karoubi filtrations, see [9]. Fact (ii) can be proved by an Eilenberg swindle argument. Fact (iii) can be checked directly. Fact (iv) is first proven in [4 for coefficients in rings, and proven for coefficients in additive categories in [8]. One has the same constructions and results for $L$-theory and details can be found in [5]. The above facts implies the following [6] [5]: 
Theorem 2.6. The $K$-theoretic $F J C_{\mathcal{A}}$ holds for $G$ if and only if the $K$-theory of $\mathcal{O}^{G}\left(E_{\mathcal{V C}} G ; \mathcal{A}\right)$ is trivial, i.e. $K_{n}\left(\mathcal{O}^{G}\left(E_{\mathcal{V C}} G ; \mathcal{A}\right)\right)=0, \forall n \in \mathbb{Z}$. The corresponding statement is true for the $L$-theoretic $F J C_{\mathcal{A}}$.

Because of this theorem, the category $\mathcal{O}^{G}\left(E_{\mathcal{V C}} G ; \mathcal{A}\right)$ is usually referred to as the obstruction category.

2.3. Some lemmas on equivariant continuous control. In this subsection, we prove some general results about the equivariantly continuously controlled condition. These results will be important in proving Theorem 3.6, which is one of the key ingredients in proving Theorem B. The proofs of some of these results and the proof of Theorem 3.6 in the next section are quite technical and readers may want to jump to Section 4 to see the proof of the main theorems first (assuming Theorem 3.6])

Now let $X$ be a $G$-CW complex and $H<G$ be a subgroup. Then $X$ is also an $H$-CW complex in a natural way. Thus we can consider $\mathcal{E}_{G c c}^{X}$ and $\mathcal{E}_{H c c}^{X}$. In the following several lemmas we study the relation between these two control conditions.

Lemma 2.7. $\mathcal{E}_{G c c}^{X} \subseteq \mathcal{E}_{H c c}^{X}$ if one of the following holds:

(i) The action of $G$ on $X$ is free.

(ii) $H<G$ is of finite index.

Proof. (i) This is obvious.

(ii) The only nontrivial part is (i) of Definition 2.2. But this can be easily shown, by noting $\bigcap_{g \in G_{x}} g U$ is a $G_{x}$-invariant neighborhood of $(x, \infty)$ in $X \times[1, \infty]$ for any $H_{x}$-invariant neighborhood $U$ of $(x, \infty)$ in $X \times[1, \infty]$, since $\left[G_{x}: H_{x}\right] \leq[G: H]<\infty$.

Lemma 2.8. If $H$ is a normal subgroup of $G$, then for any $E \in \mathcal{E}_{H c c}^{X}$ and $g \in G$, we have $g E \in \mathcal{E}_{H c c}^{X}$.

Proof. Since $H$ is a normal subgroup of $G$, one sees easily that $H \cdot g E=g E$, hence $g E$ is $H$ invariant. One also easily sees $g E$ is symmetric, bounded in the $[1, \infty)$ direction. Now for any $x \in X$, any $H_{x}$-invariant neighborhood $U$ of $(x, \infty)$ in $X \times[1, \infty]$, we consider $g^{-1} x$. Since $H<G$

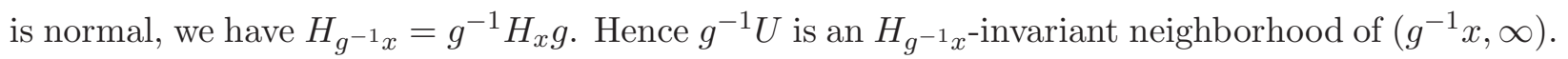

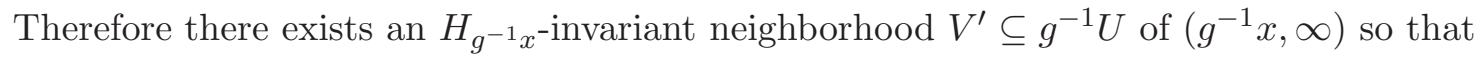

$$
\left(\left(g^{-1} U\right)^{c} \times V^{\prime}\right) \cap E=\emptyset
$$

Hence

$$
\left(U^{c} \times g V^{\prime}\right) \cap g E=\emptyset
$$

This completes the proof by letting $V=g V^{\prime}$ and by noting $V$ is $H_{x}$-invariant.

The proof of part (iv) in the proof of the following lemma is motivated from the proof of [4, Lemma 3.3]. 
Lemma 2.9. Let $E \in \mathcal{E}_{H c c}^{X}$. For any compact subset $K \subseteq X$, define

$$
E^{\prime}=\left\{(x, s) \times\left(x^{\prime}, s^{\prime}\right) \mid \exists g, g^{\prime} \in G \text { s.t. } g^{-1} g^{\prime} \in H, g^{-1} x, g^{\prime-1} x^{\prime} \in K,\left(g^{-1} x, s\right) \times\left(g^{-1} x^{\prime}, s^{\prime}\right) \in E\right\}
$$

Then $E^{\prime} \in \mathcal{E}_{G c c}^{X}$.

Proof. (i) $E^{\prime}$ is symmetric: suppose $(x, s) \times\left(x^{\prime}, s^{\prime}\right) \in E^{\prime}$, then we have $g, g^{\prime} \in G$ with the properties in the definition of $E^{\prime}$. Now since $E$ is symmetric, we have $\left(g^{-1} x^{\prime}, s^{\prime}\right) \times\left(g^{-1} x, s\right) \in E$. Since $E$ is $H$-invariant and $g^{-1} g^{\prime} \in H$, we get $\left(g^{\prime-1} x^{\prime}, s^{\prime}\right) \times\left(g^{\prime-1} x, s\right) \in E$. This shows $g^{\prime}, g$ fullfill the properties for the pair $\left(x^{\prime}, s^{\prime}\right) \times(x, s)$ in the definition of $E^{\prime}$. Hence $E^{\prime}$ is symmetric;

(ii) $E^{\prime}$ is $G$-invariant: suppose $(x, s) \times\left(x^{\prime}, s^{\prime}\right) \in E^{\prime}$ and $l \in G$. Let $g, g^{\prime}$ be as before. One easily verifies that $l g, l g^{\prime}$ fulfill the properties in the definition of $E^{\prime}$ for $(l x, s) \times\left(l x^{\prime}, s^{\prime}\right)$. Hence $(l x, s) \times\left(l x^{\prime}, s^{\prime}\right) \in E^{\prime}$, which shows $E^{\prime}$ is $G$-invariant;

(iii) Bounded control in the $s$-direction: this is clear since $E$ is controlled;

(iv) $E^{\prime}$ is $G$-equivariantly continuously controlled: suppose not, then there exists $x_{0} \in X$, a $G_{x_{0}}$-invariant open neighborhood $U$ of $x_{0} \in X$ and $r>0$, such that for all $G_{x_{0}}$-invariant open neighborhood $V \subseteq U$ of $x_{0}$ in $X$ and all $l>r$, we have

$$
(U \times(r, \infty])^{c} \times(V \times(l, \infty]) \cap E^{\prime} \neq \emptyset
$$

Now since $X$ is a G-CW complex, the Slice theorem (see [4, Proposition 3.4]) applies, so that we can find a descending sequence $\left\{V^{k}\right\}_{k \in \mathbb{N}}$ of open neighborhoods of $x_{0}$ in $X$ with the following property:

(a) Each $V^{k}$ is $G_{x_{0}}$-invariant;

(b) $g V^{k} \cap V^{k}=\emptyset$ if $g \notin G_{x_{0}}$;

(c) $\bigcap_{k \geq 1} \overline{G \cdot V^{k}}=G \cdot x_{0}$.

We may assume $V^{k} \subseteq U, \forall k \in \mathbb{N}$. Hence

$$
\left[(U \times(r, \infty])^{c} \times\left(V^{k} \times(k+r, \infty]\right)\right] \cap E^{\prime} \neq \emptyset
$$

thus we can find a sequence

$$
\left(x_{k}, s_{k}\right) \times\left(x_{k}^{\prime}, s_{k}^{\prime}\right) \in\left[(U \times(r, \infty])^{c} \times\left(V^{k} \times(k+r, \infty]\right)\right] \cap E^{\prime}
$$

By the definition of $E^{\prime}$, there exist $g_{k}, g_{k}^{\prime}$ such that

$$
g_{k}^{-1} g_{k}^{\prime} \in H, g_{k}^{-1} x_{k}, g^{\prime}{ }_{k}^{-1} x_{k}^{\prime} \in K,\left(g_{k}^{-1} x_{k}, s_{k}\right) \times\left(g_{k}^{-1} x_{k}^{\prime}, s_{k}^{\prime}\right) \in E
$$

Since $E$ is $H$-invariant and $g_{k}^{-1} g_{k}^{\prime} \in H$, the above also implies

$$
\left(g_{k}^{\prime-1} x_{k}, s_{k}\right) \times\left(g_{k}^{\prime-1} x_{k}^{\prime}, s_{k}^{\prime}\right) \in E
$$

Now since $K$ is compact, by passing to a subsequence, we may assume $g^{\prime}{ }_{k}{ }^{-1} x_{k}^{\prime} \rightarrow y$. Now since $x_{k}^{\prime} \in V^{k}$, we have for every $n \in \mathbb{N}$ and all $k>n, g^{\prime}{ }_{k}{ }^{-1} x^{\prime}{ }_{k} \in G \cdot V^{k} \subseteq G \cdot V^{n}$, this implies $y \in \overline{G \cdot V^{n}}$ for all $n$, thus $y \in \bigcap_{n \geq 1} \overline{G \cdot V^{n}}=G \cdot x_{0}$. Hence there is $g \in G$ so that $y=g x_{0}$. Therefore 
$g^{\prime}{ }_{k}{ }^{-1} x_{k}^{\prime} \rightarrow y=g x_{0}$, so $g^{-1} g^{\prime}{ }_{k}{ }^{-1} x_{k}^{\prime} \rightarrow x_{0} \in V^{1}$. Thus when $k$ is large enough $g^{-1} g^{\prime}{ }_{k}^{-1} \in G_{x_{0}}$. Note that $s_{k}^{\prime} \rightarrow \infty$, hence by 2.7 and the control condition in the $s$-direction, we have $s_{k} \rightarrow \infty$. Now by 2.5, when $k$ is large enough, $x_{k} \notin U$, hence $g^{-1} g^{\prime}{ }_{k}{ }^{-1} x_{k} \notin U$ since $g^{-1} g^{\prime}{ }^{-1} \in G_{x_{0}}$ and $U$ is $G_{x_{0}}$-invariant. Thus when $k$ is large enough, $g^{\prime}{ }^{-1} x_{k} \notin g U$.

Now consider $g U$, it is $G_{y^{-}}$-invariant since $y=g x_{0}$ and $U$ is $G_{x_{0}}$-invariant. In particular, $g U$ is $H_{y^{-}}$ invariant. Now because $E$ is $H$-equivariantly continuously controlled, we can find an $H_{y}$-invariant open neighborhood $W \subseteq g U$ of $y=g x_{0}$ in $X$ and $N>r$ such that

$$
\left[(g U \times(r, \infty])^{c} \times(W \times(N, \infty])\right] \cap E=\emptyset
$$

However, on the one hand, $\left(g_{k}^{\prime-1} x_{k}, s_{k}\right) \times\left(g_{k}^{\prime-1} x_{k}^{\prime}, s_{k}^{\prime}\right) \in E$ for all $k$ by 2.7, while on the other hand, when $k$ is large enough, we showed $g^{\prime}{ }_{k}{ }^{-1} x_{k} \notin g U, g^{\prime}{ }_{k}{ }^{-1} x_{k}^{\prime} \rightarrow y \in W$ and $s_{k}, s_{k}^{\prime} \rightarrow \infty$, so we also have $\left(g_{k}^{\prime-1} x_{k}, s_{k}\right) \times\left(g_{k}^{\prime-1} x_{k}^{\prime}, s_{k}^{\prime}\right) \in(g U \times(r, \infty])^{c} \times(W \times(N, \infty])$. Thus when $k$ is large enough, we have

$$
\left(g_{k}^{\prime-1} x_{k}, s_{k}\right) \times\left(g_{k}^{\prime-1} x_{k}^{\prime}, s_{k}^{\prime}\right) \in\left[(g U \times(r, \infty])^{c} \times(W \times(N, \infty])\right] \cap E
$$

This contradicts to 2.8 and we complete the proof.

\section{Equivalence of two CATEgories}

The major goal of this section is to prove Theorem 3.6. Let us firstly make some preparations. Let $\mathcal{A}$ be a right $G$-additive category. For any left $G$-set $X$, Bartels and Reich [8] defined a new additive category $\mathcal{A} *_{G} X$. The special case when $X=p t$ will be important in our treatment and we denote it by $\mathcal{A}_{\alpha}[G]$, where $\alpha$ denotes the right $G$-action on $\mathcal{A}$. We now recall its definition here.

Definition 3.1. ([8, Definition 2.1]) Objects of the category $\mathcal{A}_{\alpha}[G]$ are the same as the objects of $\mathcal{A}$. A morphism $\phi: A \rightarrow B$ from $A$ to $B$ in $\mathcal{A}_{\alpha}[G]$ is a formal sum $\phi=\sum_{g \in G} \phi^{g} \cdot g$, where $\phi^{g}: A \rightarrow g^{*} B$ is a morphism in $\mathcal{A}$ and there are only finitely many $g \in G$ with $\phi^{g} \neq 0$. Addition of morphisms is defined in the obvious way. Composition of morphisms is defined as follows: let

$\phi=\sum_{k \in G} \phi^{k} \cdot k: A \rightarrow B$ be a morphism from $A$ to $B$ and $\psi=\sum_{h \in G} \psi^{h} \cdot h: B \rightarrow C$ be a morphism from $B$ to $C$, their composition is given by

$$
\psi \circ \phi:=\sum_{g \in G}\left(\sum_{k, h \in G, g=h k} k^{*}\left(\psi^{h}\right) \circ \phi^{k}\right) \cdot g
$$

Now let $F$ and $G$ be two groups. Suppose there is a group homomorphism $\alpha: F \rightarrow A u t(G), f \mapsto$ $\alpha_{f}$. We then can form the associated semi-direct product $\Gamma=G \rtimes_{\alpha} F$. Every element $\gamma \in \Gamma$ can be uniquely written as $\gamma=g f$ for some $g \in G$ and $f \in F$. We have $g f g^{\prime} f^{\prime}=g \alpha_{f}\left(g^{\prime}\right) f f^{\prime}$. In particular $\alpha_{f}(g)=f g f^{-1}$. Now let $X$ be a left $\Gamma$-CW complex and $\mathcal{A}$ be a right $\Gamma$-additive category (with or without involution). Since $F$ and $G$ are subgroups of $\Gamma$, they naturally inherit actions on $X$ and 
$\mathcal{A}$. So we can consider the obstruction category $\mathcal{O}^{G}(X ; \mathcal{A})$. Recall from Definition 2.3 that

$$
\begin{gathered}
\mathcal{O}^{\Gamma}(X ; \mathcal{A})=\mathcal{C}^{\Gamma}\left(\Gamma \times X \times[1, \infty),(p \times p)^{-1} \mathcal{E}_{\Gamma c c}^{X} \cap(r \times r)^{-1} \mathcal{E}_{\Gamma}, q^{-1} \mathcal{F}_{\Gamma c} ; \mathcal{A}\right) \\
\mathcal{O}^{G}(X ; \mathcal{A})=\mathcal{C}^{G}\left(G \times X \times[1, \infty),(p \times p)^{-1} \mathcal{E}_{G c c}^{X} \cap(r \times r)^{-1} \mathcal{E}_{G}, q^{-1} \mathcal{F}_{G c} ; \mathcal{A}\right)
\end{gathered}
$$

In what follows, we first show in Lemma 3.2 that the actions of $F$ on $G, X$ and $\mathcal{A}$ induce a right $F$-action on the additive category $\mathcal{O}^{G}(X ; \mathcal{A}$ ) (which, by abuse of notation, will also be denoted by $\alpha)$. We then can form the additive category $\mathcal{O}^{G}(X ; \mathcal{A})_{\alpha}[F]$. We prove in Theorem 3.6 that the two additive categories, $\mathcal{O}^{\Gamma}(X ; \mathcal{A})$ and $\mathcal{O}^{G}(X ; \mathcal{A})_{\alpha}[F]$, are equivalent under some assumptions. In particular, these assumptions are satisfied if $F$ is finite or if the action of $\Gamma$ on $X$ is free.

In the proofs of Lemma 3.2 and Theorem 3.6, we will check everything very carefully. One might want to jump to Remark 3.4 first for a simple interpretation of the somewhat complicated formulas in the lemma below.

Lemma 3.2. Let $f \in F$. For any objects $A, B$ and morphism $\phi: A \rightarrow B$ in $\mathcal{O}^{G}(X ; \mathcal{A})$, the formulas

$$
\begin{gathered}
\left(\alpha^{f} A\right)_{(g, x, s)}:=f^{*}\left(A_{\left(\alpha_{f}(g), f x, s\right)}\right) \\
\left(\alpha^{f} \phi\right)_{\left(g^{\prime}, x^{\prime}, s^{\prime}\right),(g, x, s)}:=f^{*}\left(\phi_{\left(\alpha_{f}\left(g^{\prime}\right), f x^{\prime}, s^{\prime}\right),\left(\alpha_{f}(g), f x, s\right)}\right)
\end{gathered}
$$

where $(g, x, s),\left(g^{\prime}, x^{\prime}, s^{\prime}\right) \in G \times X \times[1, \infty)$, define an additive functor $\alpha^{f}: \mathcal{O}^{G}(X ; \mathcal{A}) \rightarrow \mathcal{O}^{G}(X ; \mathcal{A})$. Moreover, the assignment $f \mapsto \alpha^{f}$ defines a right $F$-action on the obstruction category $\mathcal{O}^{G}(X ; \mathcal{A})$.

Proof. The major part is to check for each $f \in F, \alpha^{f}: \mathcal{O}^{G}(X ; \mathcal{A}) \rightarrow \mathcal{O}^{G}(X ; \mathcal{A})$ is a well-defined additive functor. As soon as this is done, it is easy to see $\alpha$ defines a right $F$-action on $\mathcal{O}^{G}(X ; \mathcal{A})$. We will omit the $s$-component in places where it is not important for the proof.

(i) $\alpha^{f} A$ is $G$-invariant: for any $l \in G,(g, x) \in G \times X$, we have

$$
\begin{aligned}
\left(l^{*}\left(\alpha^{f} A\right)\right)_{(g, x)} & =l^{*}\left(\left(\alpha^{f} A\right)_{(l g, l x)}\right) \\
& =l^{*}\left(f^{*}\left(A_{\left(\alpha_{f}(l g), f l x\right)}\right)\right) \\
& =(f l)^{*}\left(A_{\left(\alpha_{f}(l g), f l x\right)}\right) \\
& =\left(\alpha_{f}(l) f\right)^{*}\left(A_{\left(\alpha_{f}(l g), f l x\right)}\right) \\
& =f^{*}\left(\alpha_{f}(l)^{*}\left(A_{\left(\alpha_{f}(l g), f l x\right)}\right)\right) \\
& =f^{*}\left(\left(\alpha_{f}(l)^{*} A\right)_{\left(\alpha_{f}(g), \alpha_{f}\left(l^{-1}\right) f l x\right)}\right) \\
& =f^{*}\left(A_{\left(\alpha_{f}(g), \alpha_{f}\left(l^{-1}\right) f l x\right)}\right) \\
& =f^{*}\left(A_{\left(\alpha_{f}(g), f x\right)}\right) \\
& =\left(\alpha^{f} A\right)_{(g, x)} \quad \text { Since } A \text { is } G \text {-invariant }
\end{aligned}
$$

Thus $l^{*}\left(\alpha^{f} A\right)=\alpha^{f} A$ for all $l \in G$. This proves $\alpha^{f} A$ is $G$-invariant for all $f \in F$. 
(ii) $\alpha^{f} \phi$ is $G$-invariant: for any $l \in G,(g, x),\left(g^{\prime}, x^{\prime}\right) \in G \times X$, we have

$$
\begin{aligned}
\left(l^{*}\left(\alpha^{f} \phi\right)\right)_{\left(g^{\prime}, x^{\prime}\right),(g, x)} & =l^{*}\left[\left(\alpha^{f} \phi\right)_{\left(l g^{\prime}, l x^{\prime}\right),(l g, l x)}\right] \\
& =l^{*}\left[f^{*}\left(\phi_{\left(\alpha_{f}\left(l g^{\prime}\right), f l x^{\prime}\right),\left(\alpha_{f}(l g), f l x\right)}\right]\right. \\
& =(f l)^{*}\left[\phi_{\left(\alpha_{f}\left(l g^{\prime}\right), f l x^{\prime}\right),\left(\alpha_{f}(l g), f l x\right)}\right] \\
& =\left(\alpha_{f}(l) f\right)^{*}\left[\phi_{\left(\alpha_{f}\left(l g^{\prime}\right), f l x^{\prime}\right),\left(\alpha_{f}(l g), f l x\right)}\right] \\
& =f^{*}\left[\alpha_{f}(l)^{*}\left(\phi_{\left(\alpha_{f}\left(l g^{\prime}\right), f l x^{\prime}\right),\left(\alpha_{f}(l g), f l x\right)}\right)\right] \\
& =f^{*}\left[\left(\alpha_{f}(l)^{*} \phi\right)_{\left(\alpha_{f}\left(g^{\prime}\right), \alpha_{f}\left(l^{-1}\right) f l x^{\prime}\right),\left(\alpha_{f}(g), \alpha_{f}\left(l^{-1}\right) f l x\right)}\right] \\
& =f^{*}\left[\phi_{\left(\alpha_{f}\left(g^{\prime}\right), f x^{\prime}\right),\left(\alpha_{f}(g), f x\right)}\right] \\
& =\left(\alpha^{f} \phi\right)_{\left(g^{\prime}, x^{\prime}\right),(g, x)}
\end{aligned}
$$

Thus $l^{*}\left(\alpha^{f} \phi\right)=\alpha^{f} \phi$ for all $l \in G$. This proves $\alpha^{f} \phi$ is $G$-invariant for all $f \in F$.

(iii) $\alpha^{f}\left(i d_{A}\right)=i d_{\alpha^{f} A}$ : this is easy.

(iv) $\alpha^{f}(\phi \circ \psi)=\left(\alpha^{f} \phi\right) \circ\left(\alpha^{f} \psi\right)$ : in the following identities, we are taking sum over $(l, y) \in G \times X$. We have

$$
\begin{aligned}
{\left[\alpha^{f}(\phi \circ \psi)\right]_{\left(g^{\prime}, x^{\prime}\right),(g, x)} } & =f^{*}\left[(\phi \circ \psi)_{\left(\alpha_{f}\left(g^{\prime}\right), f x^{\prime}\right),\left(\alpha_{f}(g), f x\right)}\right] \\
& =f^{*}\left[\phi_{\left(\alpha_{f}\left(g^{\prime}\right), f x^{\prime}\right),(l, y)} \circ \psi_{(l, y),\left(\alpha_{f}(g), f x\right)}\right] \\
& =f^{*}\left[\phi_{\left(\alpha_{f}\left(g^{\prime}\right), f x^{\prime}\right),(l, y)} \circ f^{*}\left[\psi_{(l, y),\left(\alpha_{f}(g), f x\right)}\right]\right. \\
& =\left(\alpha^{f} \phi\right)_{\left(g^{\prime}, x^{\prime}\right),\left(\alpha_{f-1}(l), f^{-1} y\right)} \circ\left(\alpha^{f} \psi\right)_{\left(\alpha_{f-1}(l), f^{-1} y\right),(g, x)} \\
& =\left[\left(\alpha^{f} \phi\right) \circ\left(\alpha^{f} \psi\right)\right]_{\left(g^{\prime}, x^{\prime}\right),(g, x)}
\end{aligned}
$$

The last identity holds because $\left(\alpha_{f^{-1}}(l), f^{-1} y\right)$ runs over $G \times X$ as $(l, y)$ runs over $G \times X$. We thus get $\alpha^{f}(\phi \circ \psi)=\left(\alpha^{f} \phi\right) \circ\left(\alpha^{f} \psi\right)$

(v) Additivity: this is easy.

(vi) Object support: we have to show $\left\{g^{-1} x \mid(g, x, s) \in \operatorname{supp}\left(\alpha^{f} A\right)\right\}$ is contained in a compact subset of $X$. Note that $\operatorname{supp}\left(\alpha^{f} A\right)=\left\{(g, x, s) \mid\left(\alpha_{f}(g), f x, s\right) \in \operatorname{supp}(A)\right\}$. By definition, there exists a compact subset $K \subseteq X$, so that $\left\{\alpha_{f}\left(g^{-1}\right) f x=f g^{-1} x \mid\left(\alpha_{f}(g), f x, s\right) \in \operatorname{supp}(A)\right\} \subseteq K$. This implies $\left\{g^{-1} x \mid(g, x, s) \in \operatorname{supp}\left(\alpha^{f} A\right)\right\}=\left\{g^{-1} x \mid\left(\alpha_{f}(g), f x, s\right) \in \operatorname{supp}(A)\right\} \subseteq f^{-1} \cdot K$, which completes the proof since $f^{-1} \cdot K \subseteq X$ is compact.

(vii) Morphism support: by definition, there exists $E \in \mathcal{E}_{G c c}^{X}$, so that the projection of $\operatorname{supp}(\phi)$ in $(X \times[1, \infty))^{2}$ is contained in $E$. Now by definition of $\alpha^{f} \phi$, the projection of $\operatorname{supp}\left(\alpha^{f} \phi\right)$ in 
$(X \times[1, \infty))^{2}$ is contained in

$$
E^{\prime}=\left\{(x, s) \times\left(x^{\prime}, s^{\prime}\right) \mid(f x, s) \times\left(f x^{\prime}, s^{\prime}\right) \in E\right\}=f^{-1} \cdot E
$$

Now since $G<\Gamma$ is a normal subgroup, by Lemma 2.8, we have $E^{\prime}=f^{-1} \cdot E \in \mathcal{E}_{G c c}^{X}$. Hence $\alpha^{f} \phi$ respects the support condition in the $X \times[1, \infty)$-direction. But clearly it respects the support condition in the $G$-direction since $\alpha_{f}: G \rightarrow G$ is an automorphism. Therefore $\alpha^{f} \phi$ respects the morphism support condition.

Now if $\mathcal{A}$ has an involution, then $\alpha^{f}$ commutes with the induced involution on $\mathcal{O}^{G}(X ; \mathcal{A})$. This together with the above verification work shows that for each $f \in F, \alpha^{f}$ is a well-defined additive functor from $\mathcal{O}^{G}(X ; \mathcal{A})$ to itself.

Finally $\alpha^{e}$ is the identity functor, where $e \in F$ is the trivial element and for $f, f^{\prime} \in F$, we have

$$
\begin{aligned}
\left(\alpha^{\left.f f^{\prime} A\right)_{(g, x)}}\right. & =\left(f f^{\prime}\right)^{*}\left[A_{\left(\alpha_{f f^{\prime}}(g), f f^{\prime} x\right)}\right] \\
& =\left(f^{\prime}\right)^{*}\left[f^{*}\left(A_{\left(\alpha_{f f^{\prime}}(g), f f^{\prime} x\right)}\right)\right] \\
& =\left(f^{\prime}\right)^{*}\left[\left(\alpha^{f} A\right)_{\left.\left(\alpha_{f^{\prime}}(g)\right), f^{\prime} x\right)}\right] \\
& =\left(\alpha^{f^{\prime}}\left(\alpha^{f} A\right)\right)_{(g, x)}
\end{aligned}
$$

Therefore $\alpha^{f f^{\prime}}=\alpha^{f^{\prime}} \alpha^{f}$ on objects. Similarly this identity holds on morphisms. Hence $f \mapsto \alpha^{f}$ defines a right $F$-action on $\mathcal{O}^{G}(X ; \mathcal{A})$. We thus complete the proof of the lemma.

Remark 3.3. We have used $\alpha$ to denote both the action of $F$ on $G$ and the action of $F$ on $\mathcal{O}^{G}(X ; \mathcal{A})$. Note however that the former action is from left while the later action is from right. It will be clear from the context which action $\alpha$ stands for.

Remark 3.4. A convenient way to interpret the defining formulas in the above Lemma is as follows: for any object $A$ in $\mathcal{O}^{G}(X ; \mathcal{A})$, it is completely determined by its values at points of the form $(e, x, s) \in G \times X \times[1, \infty)$, where $e \in G$ is the identity element. But $(e, x, s)$ is a point in $\Gamma \times X \times[1, \infty)$, using the $\Gamma$-action on $\Gamma \times X \times[1, \infty), A_{(e, x, s)}$ can be used to define an object $\tau A$ living over $\Gamma \times X \times[1, \infty)$, i.e. by defining $(\tau A)_{(\gamma, x, s)}:=\left(\gamma^{-1}\right)^{*}\left(A_{\left(e, \gamma^{-1} x, s\right)}\right)(\tau A$ is actually an object in $\mathcal{O}^{\Gamma}(X ; \mathcal{A})$ that we will show shortly). Then $A$ is just the restriction of $\tau A$ to $G \times X \times[1, \infty)$. Note for any $f \in F$, the map $(\gamma, x, s) \mapsto(\gamma f, x, s)$ is a $\Gamma$-equivariant self-homeomorphism of $\Gamma \times X \times[1, \infty)$, it thus induces an automorphism of $\mathcal{O}^{\Gamma}(X ; \mathcal{A})$. Denote this automorphism by $\alpha^{f}$, then $\left(\alpha^{f}(\tau A)\right)_{(\gamma, x, s)}=(\tau A)_{\left(\gamma f^{-1}, x, s\right)}=f^{*}\left((\tau A)_{\left(f \gamma f^{-1}, f x, s\right)}\right)$, the second equality is due to the $\Gamma$-invariance of $\tau A$. This formula restricts to $G \times X \times[1, \infty)$ is the defining formula on objects in the above lemma. From this point of view, Lemma 3.2 is morally apparent.

Remark 3.5. The naive definition $\left(\alpha^{f} A\right)_{(g, x, s)}:=A_{\left(\alpha_{f}(g), x, s\right)}$ doesn't work since the map $(g, x, s) \mapsto$ $\left(\alpha_{f}(g), x, s\right)$ is not $G$-equivariant. 
We can now form the category $\mathcal{O}^{G}(X ; \mathcal{A})_{\alpha}[F]$ and are going to show $\mathcal{O}^{\Gamma}(X ; \mathcal{A})$ and $\mathcal{O}^{G}(X ; \mathcal{A})_{\alpha}[F]$ are equivalent as additive categories (with involution) under some conditions. Forgetting about the control conditions, this is then not very hard to see intuitively. The one-one correspondence between objects of these two categories has already been explained in Remark 3.4. Let us focus on morphisms. By $\Gamma$-invariance, every morphism $\phi: A \rightarrow B$ in $\mathcal{O}^{\Gamma}(X ; \mathcal{A})$ is uniquely determined by its values on $(e, x, s) \times\left(\gamma^{\prime}, x^{\prime}, s^{\prime}\right)$, i.e. by $\phi_{\left(\gamma^{\prime}, x^{\prime}, s^{\prime}\right),(e, x, s)}: A_{(e, x, s)} \rightarrow B_{\left(\gamma^{\prime}, x^{\prime}, s^{\prime}\right)}$. They can be grouped into families $\left\{\phi_{\left(g f, x^{\prime}, s^{\prime}\right),(e, x, s)} \mid g \in G\right\}, f \in F$. Due to the morphism control condition in the $\Gamma$-direction, see Definition 2.3, there are only finitely many $f \in F$ whose corresponding family is non-trivial. For each of such $f$, the family indexed by $f$ determines a morphism $\phi^{f}: A \rightarrow \alpha^{f} B$ in $\mathcal{O}^{G}(X ; \mathcal{A})$, here we are viewing objects $A$ and $B$ as objects in $\mathcal{O}^{G}(X ; \mathcal{A})$ by restriction to $G \times X \times[1, \infty)$. The map $\phi \rightarrow \sum_{f \in F} \phi^{f} \cdot f$ then gives a one-one correspondence between morphisms of these two category.

Theorem 3.6. Let $\Gamma=G \rtimes_{\alpha} F$ be as before. Then for any $\Gamma-C W$ complex $X$ and any additive category (with involution) $\mathcal{A}$ with a right $\Gamma$-action, the two additive categories (with involutions) $\mathcal{O}^{\Gamma}(X ; \mathcal{A})$ and $\mathcal{O}^{G}(X ; \mathcal{A})_{\alpha}[F]$ are equivalent provided $\mathcal{E}_{\Gamma c c}^{X} \subseteq \mathcal{E}_{G c c}^{X}$. In particular, they are equivalent if $F$ is finite or if the action of $\Gamma$ on $X$ is free.

Proof. Define a functor $\tau: \mathcal{O}^{G}(X ; \mathcal{A})_{\alpha}[F] \rightarrow \mathcal{O}^{\Gamma}(X ; \mathcal{A})$ as follows:

on objects:

$$
(\tau A)_{(\gamma, x, s)}:=f^{*}\left(A_{\left(\alpha_{f}(g), f x, s\right)}\right)=\left(\alpha^{f} A\right)_{(g, x, s)}
$$

where $(\gamma, x, s) \in \Gamma \times X \times[1, \infty)$ and $g \in G, f \in F$ are the unique elements so that $\gamma=g f^{-1}$. One can easily check that $(\tau A)_{(\gamma, x, s)}=\left(\gamma^{-1}\right)^{*}\left(A_{\left(e, \gamma^{-1} x, s\right)}\right)$, from which we conclude $\tau A$ is $\Gamma$-invariant.

on morphisms: Let $\phi=\sum_{f \in F} \phi^{f} \cdot f: A \rightarrow B$ be a morphism in $\mathcal{O}^{G}(X ; \mathcal{A})_{\alpha}[F]$, where $\phi^{f}: A \rightarrow \alpha^{f} B$ is a morphism in $\mathcal{O}^{G}(X ; \mathcal{A})$. By definition, there are only finitely many $f \in F$ with $\phi^{f} \neq 0$.

Define

$$
\tau \phi: \tau A \rightarrow \tau B
$$

to be the sum $\sum_{f \in F} \tau_{f} \phi^{f}$, where

$$
\tau_{f} \phi^{f}: \tau A \rightarrow \tau B
$$

is defined as follows:

$$
\left(\tau_{f} \phi^{f}\right)_{\left(\gamma^{\prime}, x^{\prime}, s^{\prime}\right),(\gamma, x, s)}:= \begin{cases}0 & \text { if } \gamma^{-1} \gamma^{\prime} \notin G f^{-1} \\ \left(\gamma^{-1}\right)^{*}\left(\phi_{\left(\gamma^{-1} \gamma^{\prime} f, \gamma^{-1} x^{\prime}, s^{\prime}\right),\left(e, \gamma^{-1} x, s\right)}^{f}\right) & \text { if } \gamma^{-1} \gamma^{\prime} \in G f^{-1}\end{cases}
$$


We have to check $\left(\gamma^{-1}\right)^{*}\left(\phi_{\left(\gamma^{-1} \gamma^{\prime} f, \gamma^{-1} x^{\prime}, s^{\prime}\right),\left(e, \gamma^{-1} x, s\right)}^{f}\right)$ is indeed a map from $(\tau A)_{(\gamma, x, s)}$ to $(\tau B)_{\left(\gamma^{\prime}, x^{\prime}, s^{\prime}\right)}$. But this is because

$$
\begin{gathered}
(\tau A)_{(\gamma, x, s)}=\left(\gamma^{-1}\right)^{*}\left(A_{\left(e, \gamma^{-1} x, s\right)}\right) \\
(\tau B)_{\left(\gamma^{\prime}, x^{\prime}, s^{\prime}\right)}=\left(\gamma^{-1}\right)^{*}\left((\tau B)_{\left(\gamma^{-1} \gamma^{\prime}, \gamma^{-1} x^{\prime}, s^{\prime}\right)}\right)=\left(\gamma^{-1}\right)^{*}\left(\left(\alpha^{f} B\right)_{\left(\gamma^{-1} \gamma^{\prime} f, \gamma^{-1} x^{\prime}, s^{\prime}\right)}\right)
\end{gathered}
$$

and

$$
\phi_{\left(\gamma^{-1} \gamma^{\prime} f, \gamma^{-1} x^{\prime}, s^{\prime}\right),\left(e, \gamma^{-1} x, s\right)}^{f}: A_{\left(e, \gamma^{-1} x, s\right)} \longrightarrow\left(\alpha^{f} B\right)_{\left(\gamma^{-1} \gamma^{\prime} f, \gamma^{-1} x^{\prime}, s^{\prime}\right)}
$$

The formulas above are motivated by the intuition explained in the paragraph preceding this theorem. We firstly show $\tau$ is well-defined, i.e. it is a genuine additive functor and respects control conditions on objects and morphisms. We then show $\tau$ actually gives an equivalence of two additive categories. In what follows, we will again omit the $s$-component in appropriate places.

(i) $\tau A$ is $\Gamma$-invariant: already checked.

(ii) $\tau \phi$ is $\Gamma$-invariant: it suffices to check for each $f \in F, \tau_{f} \phi^{f}$ is $\Gamma$-invariant. For any $l \in \Gamma$, we have

$$
\left[l^{*}\left(\tau_{f} \phi^{f}\right)\right]_{\left(\gamma^{\prime}, x^{\prime}\right),(\gamma, x)}=l^{*}\left[\left(\tau_{f} \phi^{f}\right)_{\left(l \gamma^{\prime}, l x^{\prime}\right),(l \gamma, l x)}\right]
$$

If $\gamma^{-1} \gamma^{\prime} \notin G f^{-1}$, then $(l \gamma)^{-1} l \gamma^{\prime} \notin G f^{-1}$, hence $\left[l^{*}\left(\tau_{f} \phi^{f}\right)\right]_{\left(\gamma^{\prime}, x^{\prime}\right),(\gamma, x)}=0=\left(\tau_{f} \phi^{f}\right)_{\left(\gamma^{\prime}, x^{\prime}\right),(\gamma, x)}$. If $\gamma^{-1} \gamma^{\prime} \in G f^{-1}$, then

$$
\begin{aligned}
{\left[l^{*}\left(\tau_{f} \phi^{f}\right)\right]_{\left(\gamma^{\prime}, x^{\prime}\right),(\gamma, x)} } & =l^{*}\left[\left(\tau_{f} \phi^{f}\right)_{\left(l \gamma^{\prime}, l x^{\prime}\right),(l \gamma, l x)}\right] \\
& =l^{*}\left[\left(\gamma^{-1} l^{-1}\right)^{*}\left(\phi_{\left(\gamma^{-1} \gamma^{\prime} f, \gamma^{-1} x^{\prime}\right),\left(e, \gamma^{-1} x\right)}^{f}\right)\right] \\
& =\left(\gamma^{-1}\right)^{*}\left(\phi_{\left(\gamma^{-1} \gamma^{\prime} f, \gamma^{-1} x^{\prime}\right),\left(e, \gamma^{-1} x\right)}^{f}\right) \\
& =\left(\tau_{f} \phi^{f}\right)_{\left(\gamma^{\prime}, x^{\prime}\right),(\gamma, x)}
\end{aligned}
$$

Thus $l^{*}\left(\tau_{f} \phi^{f}\right)=\tau_{f} \phi^{f}$, for all $f \in F$ and $l \in \Gamma$. This shows $\tau \phi$ is $\Gamma$-invariant.

(iii) $\tau\left(i d_{A}\right)=i d_{\tau A}$ : note $i d_{A}: A \rightarrow A$ in $\mathcal{O}^{G}(X ; \mathcal{A})_{\alpha}[F]$ is given by $i d_{A} \cdot e$. Hence

$$
\begin{aligned}
{\left[\tau\left(i d_{A}\right)\right]_{\left(\gamma^{\prime}, x^{\prime}\right),(\gamma, x)} } & = \begin{cases}0 & \text { if } \gamma^{-1} \gamma^{\prime} \notin G \\
\left(\gamma^{-1}\right)^{*}\left[\left(i d_{A}\right)_{\left(\gamma^{-1} \gamma^{\prime}, \gamma^{-1} x^{\prime}\right),\left(e, \gamma^{-1} x\right)}\right] & \text { if } \gamma^{-1} \gamma^{\prime} \in G\end{cases} \\
& = \begin{cases}0 & \text { if }(\gamma, x) \neq\left(\gamma^{\prime}, x^{\prime}\right) \\
\left(i d_{\tau A}\right)\left(\gamma^{\prime}, x^{\prime}\right),(\gamma, x) & \text { if }(\gamma, x)=\left(\gamma^{\prime}, x^{\prime}\right)\end{cases}
\end{aligned}
$$


(iv) $\tau(\psi \circ \phi)=(\tau \psi) \circ(\tau \phi)$ : Let $\phi=\sum_{f \in F} \phi^{f} \cdot f: A \rightarrow B$ and $\psi=\sum_{h \in F} \psi^{h} \cdot h: B \rightarrow C$ be two morphisms in $\mathcal{O}^{G}(X ; \mathcal{A})_{\alpha}[F]$. We have

$$
\tau(\psi \circ \phi)=\tau\left(\sum_{k \in F}\left(\sum_{h f=k}\left(\alpha^{f} \psi^{h}\right) \circ \phi^{f}\right) \cdot k\right)=\sum_{k \in F} \sum_{h f=k} \tau_{k}\left(\left(\alpha^{f} \psi^{h}\right) \circ \phi^{f}\right)
$$

and

$$
\tau(\psi) \circ \tau(\phi)=\left(\sum_{h \in F} \tau_{h} \psi^{h}\right) \circ\left(\sum_{f \in F} \tau_{f} \phi^{f}\right)=\sum_{k \in F} \sum_{h f=k}\left(\tau_{h} \psi^{h}\right) \circ\left(\tau_{f} \phi^{f}\right)
$$

So it suffices to show $\tau_{k}\left(\left(\alpha^{f} \psi^{h}\right) \circ \phi^{f}\right)=\left(\tau_{h} \psi^{h}\right) \circ\left(\tau_{f} \phi^{f}\right)$ when $k=h f$.

Now by definition, when $\gamma^{-1} \gamma^{\prime} \notin G k^{-1}$, we have

$$
\left[\tau_{k}\left(\left(\alpha^{f} \psi^{h}\right) \circ \phi^{f}\right)\right]_{\left(\gamma^{\prime}, x^{\prime}\right),(\gamma, x)}=0
$$

and

$$
\left[\left(\tau_{h} \psi^{h}\right) \circ\left(\tau_{f} \phi^{f}\right)\right]_{\left(\gamma^{\prime}, x^{\prime}\right),(\gamma, x)}=\left(\tau_{h} \psi^{h}\right)_{\left(\gamma^{\prime}, x^{\prime}\right),\left(\gamma^{\prime \prime}, x^{\prime \prime}\right)} \circ\left(\tau_{f} \phi^{f}\right)_{\left(\gamma^{\prime \prime}, x^{\prime \prime}\right),(\gamma, x)}
$$

where on the right hand side of the above identity, we are taking sum over $\left(\gamma^{\prime \prime}, x^{\prime \prime}\right) \in \Gamma \times X$. However a term in this sum can be non-zero only when $\gamma^{\prime \prime-1} \gamma^{\prime} \in G h^{-1}$ and $\gamma^{-1} \gamma^{\prime \prime} \in G f^{-1}$, which implies when $\gamma^{-1} \gamma^{\prime} \notin G k^{-1}$, it must be zero. This verifies $\left[\tau_{k}\left(\left(\alpha^{f} \psi^{h}\right) \circ \phi^{f}\right)\right]_{\left(\gamma^{\prime}, x^{\prime}\right),(\gamma, x)}=$ $\left[\left(\tau_{h} \psi^{h}\right) \circ\left(\tau_{f} \phi^{f}\right)\right]_{\left(\gamma^{\prime}, x^{\prime}\right),(\gamma, x)}$ when $\gamma^{-1} \gamma^{\prime} \notin G k^{-1}$.

When $\gamma^{-1} \gamma^{\prime} \in G k^{-1}$, on one hand, we have (in the following identities, we are taking sum over $\left(g^{\prime \prime}, x^{\prime \prime}\right) \in G \times X$.)

$$
\begin{aligned}
& {\left[\tau_{k}\left(\left(\alpha^{f} \psi^{h}\right) \circ \phi^{f}\right)\right]_{\left(\gamma^{\prime}, x^{\prime}\right),(\gamma, x)}} \\
& \left.=\left(\gamma^{-1}\right)^{*}\left[\left(\alpha^{f} \psi^{h}\right) \circ \phi^{f}\right)_{\left(\gamma^{-1} \gamma^{\prime} k, \gamma^{-1} x^{\prime}\right),\left(e, \gamma^{-1} x\right)}\right] \\
& =\left(\gamma^{-1}\right)^{*}\left[\left(\alpha^{f} \psi^{h}\right)_{\left(\gamma^{-1} \gamma^{\prime} k, \gamma^{-1} x^{\prime}\right),\left(g^{\prime \prime}, x^{\prime \prime}\right)} \circ \phi_{\left(g^{\prime \prime}, x^{\prime \prime}\right),\left(e, \gamma^{-1} x\right)}^{f}\right] \\
& =\left(\gamma^{-1}\right)^{*}\left[f^{*}\left(\psi_{\left(\alpha_{f}\left(\gamma^{-1} \gamma^{\prime} k\right), f \gamma^{-1} x^{\prime}\right),\left(\alpha_{f}\left(g^{\prime \prime}\right), f x^{\prime \prime}\right)}^{h}\right) \circ \phi_{\left(g^{\prime \prime}, x^{\prime \prime}\right),\left(e, \gamma^{-1} x\right)}^{f}\right] \\
& \left.=\left(\gamma^{-1}\right)^{*}\left[\left(\alpha_{f}\left(g^{\prime \prime-1}\right) f\right)^{*}\left(\psi_{\left(\alpha_{f}\left(g^{\prime \prime-1} \gamma^{-1} \gamma^{\prime} k\right), \alpha_{f}\left(g^{\prime \prime-1}\right) f \gamma^{-1} x^{\prime}\right),\left(e, \alpha_{f}\left(g^{\prime \prime-1}\right) f x^{\prime \prime}\right)}^{h} \phi_{\left(g^{\prime \prime}, x^{\prime \prime}\right),\left(e, \gamma^{-1} x\right)}^{f}\right]\right)\right] \\
& =\left(\gamma^{-1}\right)^{*}\left[\left(f g^{\prime \prime-1}\right)^{*}\left(\psi_{\left(\alpha_{f}\left(g^{\prime \prime-1} \gamma^{-1} \gamma^{\prime} k\right), f g^{\prime \prime-1} \gamma^{-1} x^{\prime}\right),\left(e, f g^{\prime \prime-1} x^{\prime \prime}\right)}^{h} \circ \phi_{\left(g^{\prime \prime}, x^{\prime \prime}\right),\left(e, \gamma^{-1} x\right)}^{f}\right)\right] \\
& =\left(f g^{\prime \prime-1} \gamma^{-1}\right)^{*}\left(\psi_{\left(\alpha_{f}\left(g^{\prime \prime-1} \gamma^{-1} \gamma^{\prime} k\right), f g^{\prime \prime-1} \gamma^{-1} x^{\prime}\right),\left(e, f g^{\prime \prime-1} x^{\prime \prime}\right)}^{h}\right) \circ\left(\gamma^{-1}\right)^{*}\left(\phi_{\left(g^{\prime \prime}, x^{\prime \prime}\right),\left(e, \gamma^{-1} x\right)}^{f}\right)
\end{aligned}
$$

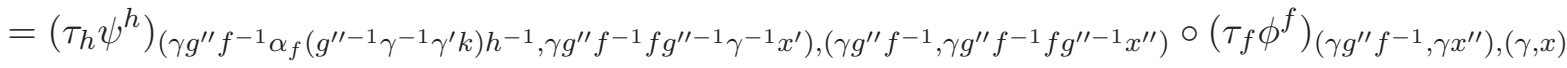

$$
\begin{aligned}
& =\left(\tau_{h} \psi^{h}\right)_{\left(\gamma^{\prime} k f^{-1} h^{-1}, x^{\prime}\right),\left(\gamma g^{\prime \prime} f^{-1}, \gamma x^{\prime \prime}\right)} \circ\left(\tau_{f} \phi^{f}\right)_{\left(\gamma g^{\prime \prime} f^{-1}, \gamma x^{\prime \prime}\right),(\gamma, x)} \\
& =\left(\tau_{h} \psi^{h}\right)_{\left(\gamma^{\prime}, x^{\prime}\right),\left(\gamma g^{\prime \prime} f^{-1}, \gamma x^{\prime \prime}\right)} \circ\left(\tau_{f} \phi^{f}\right)_{\left(\gamma g^{\prime \prime} f^{-1}, \gamma x^{\prime \prime}\right),(\gamma, x)} \\
& =\left(\tau_{h} \psi^{h}\right)_{\left(\gamma^{\prime}, x^{\prime}\right),\left(\gamma g^{\prime \prime} f^{-1}, x^{\prime \prime}\right)} \circ\left(\tau_{f} \phi^{f}\right)_{\left(\gamma g^{\prime \prime} f^{-1}, x^{\prime \prime}\right),(\gamma, x)}
\end{aligned}
$$

The last identity holds because when $x^{\prime \prime}$ runs over $X, \gamma x^{\prime \prime}$ also runs over $X$. 
Now on the other hand, we have

$$
\left[\left(\tau_{h} \psi^{h}\right) \circ\left(\tau_{f} \phi^{f}\right)\right]_{\left(\gamma^{\prime}, x^{\prime}\right),(\gamma, x)}=\left(\tau_{h} \psi^{h}\right)_{\left(\gamma^{\prime}, x^{\prime}\right),\left(\gamma^{\prime \prime}, x^{\prime \prime}\right)} \circ\left(\tau_{f} \phi^{f}\right)_{\left(\gamma^{\prime \prime}, x^{\prime \prime}\right),(\gamma, x)}
$$

Note we are taking sum over $\left(\gamma^{\prime \prime}, x^{\prime \prime}\right) \in \Gamma \times X$ on the right hand of the above identity. However only those terms with $\gamma^{-1} \gamma^{\prime \prime} \in G f^{-1}$ can be non-zero (note $\gamma^{-1} \gamma^{\prime \prime} \in G f^{-1}, \gamma^{-1} \gamma^{\prime} \in G k^{-1}$ together with $k=h f$ imply $\left.\gamma^{\prime \prime-1} \gamma^{\prime} \in G h^{-1}\right)$. So we only have to take sum over those terms with $\gamma^{\prime \prime}=$ $\gamma g^{\prime \prime} f^{-1}, g^{\prime \prime} \in G$. Hence we have

$$
\begin{aligned}
& {\left[\left(\tau_{h} \psi^{h}\right) \circ\left(\tau_{f} \phi^{f}\right)\right]_{\left(\gamma^{\prime}, x^{\prime}\right),(\gamma, x)} } \\
= & \left(\tau_{h} \psi^{h}\right)_{\left(\gamma^{\prime}, x^{\prime}\right),\left(\gamma g^{\prime \prime} f^{-1}, x^{\prime \prime}\right)} \circ\left(\tau_{f} \phi^{f}\right)_{\left(\gamma g^{\prime \prime} f^{-1}, x^{\prime \prime}\right),(\gamma, x)}
\end{aligned}
$$

Therefore $\tau_{k}\left(\left(\alpha^{f} \psi^{h}\right) \circ \phi^{f}\right)=\left(\tau_{h} \psi^{h}\right) \circ\left(\tau_{f} \phi^{f}\right)$ when $k=h f$. Thus $\tau(\psi \circ \phi)=(\tau \psi) \circ(\tau \phi)$.

(v) Additivity: this is easy.

(vi) Object support: $(\gamma, x, s) \in \operatorname{supp}(\tau A)$ if and only if $\left(e, \gamma^{-1} x, s\right) \in \operatorname{supp}(A)$. By definition, there is a compact set $K \subseteq X$, so that $\gamma^{-1} x \in K$ for all $\left(e, \gamma^{-1} x, s\right) \in \operatorname{supp}(A)$. Hence $\gamma^{-1} x \in K$ for all $(\gamma, x, s) \in \operatorname{supp}(\tau A)$. This shows $\tau A$ respects the object support condition.

(vii) Morphism support: we firstly show each $\tau_{f} \phi^{f}, f \in F$ respects the morphism support condition. By definition of $\tau_{f} \phi^{f}$, its morphism support condition in the $\Gamma$-direction is easily seen to be satisfied. Let us focus on its morphism support condition in the $X \times[1, \infty)$-direction. By definition, for $\phi^{f}: A \rightarrow \alpha^{f} B$, there exists $E \in \mathcal{E}_{G c c}^{X}$, such that the projection of $\operatorname{supp}\left(\phi^{f}\right)$ in $(X \times[1, \infty))^{2}$ is contained in $E$. By definition of $\tau_{f} \phi^{f}$ and the fact that $\operatorname{supp}(A)$ and $\operatorname{supp}\left(\alpha^{f} B\right)$ are $G$-cocompact in the $G \times X$-direction, there exists a compact subset $K \subseteq X$, such that the projection of $\operatorname{supp}\left(\tau_{f} \phi^{f}\right)$ in $(X \times[1, \infty))^{2}$ is contained in

$E^{\prime}=\left\{(x, s) \times\left(x^{\prime}, s^{\prime}\right) \mid \exists \gamma, \gamma^{\prime} \in \Gamma\right.$ s.t. $\left.\gamma^{-1} \gamma^{\prime} \in G f^{-1}, \gamma^{-1} x, f^{-1} \gamma^{\prime-1} x^{\prime} \in K,\left(\gamma^{-1} x, s\right) \times\left(\gamma^{-1} x^{\prime}, s^{\prime}\right) \in E\right\}$

Replacing $\gamma^{\prime}$ by $\gamma^{\prime} f^{-1}$, one sees

$$
E^{\prime}=\left\{(x, s) \times\left(x^{\prime}, s^{\prime}\right) \mid \exists \gamma, \gamma^{\prime} \in \Gamma \text { s.t. } \gamma^{-1} \gamma^{\prime} \in G, \gamma^{-1} x, \gamma^{\prime-1} x^{\prime} \in K,\left(\gamma^{-1} x, s\right) \times\left(\gamma^{-1} x^{\prime}, s^{\prime}\right) \in E\right\}
$$

But by Lemma 2.9, $E^{\prime} \in \mathcal{E}_{\Gamma c c}^{X}$. This shows, for each $f \in F, \tau_{f} \phi^{f}$ respects the morphism support condition for morphisms in $\mathcal{O}^{\Gamma}(X ; \mathcal{A})$. Therefore $\tau \phi=\sum_{f \in F} \tau_{f} \phi^{f}$ respects the morphism support condition in $\mathcal{O}^{\Gamma}(X ; \mathcal{A})$ since $\operatorname{supp}(\tau \phi)$ is contained in the finite union $\bigcup_{f \in F} \operatorname{supp}\left(\tau_{f} \phi^{f}\right)$.

We now complete the verification work that $\tau$ is a well-defined functor from $\mathcal{O}^{G}(X ; \mathcal{A})_{\alpha}[F]$ to $\mathcal{O}^{\Gamma}(X ; \mathcal{A})$. Next we show it is an equivalence of additive categories (with involution). 
(a) $\tau$ is full on objects: for any object $A$ in $\mathcal{O}^{\Gamma}(X ; \mathcal{A})$, let $\operatorname{Res} A$ denote its restriction to $G \times$ $X \times[1, \infty)$, i.e. $(\operatorname{Res} A)_{(g, x, s)}:=A_{(g, x, s)},(g, x, s) \in G \times X \times[1, \infty)$. Clearly $\operatorname{Res} A$ is an object in $\mathcal{O}^{G}(X ; \mathcal{A})_{\alpha}[F]$. One also easily sees that $\tau(\operatorname{Res} A)=A$.

(b) $\tau$ is full on morphisms: for any objects $A, B$ in $\mathcal{O}^{G}(X ; \mathcal{A})_{\alpha}[F]$, morphism $\Phi: \tau A \rightarrow \tau B$ in $\mathcal{O}^{\Gamma}(X ; \mathcal{A})$, and $f \in F$, define

$$
\phi^{f}: A \rightarrow \alpha^{f} B
$$

by defining

$$
\phi_{\left(g^{\prime}, x^{\prime}, s^{\prime}\right),(g, x, s)}^{f}:=\Phi_{\left(g^{\prime} f^{-1}, x^{\prime}, s^{\prime}\right),(g, x, s)}
$$

Note since

$$
\Phi_{\left(g^{\prime} f^{-1}, x^{\prime}, s^{\prime}\right),(g, x, s)}:(\tau A)_{(g, x, s)}=A_{(g, x, s)} \longrightarrow(\tau B)_{\left(g^{\prime} f^{-1}, x^{\prime}, s^{\prime}\right)}=\left(\alpha^{f} B\right)_{\left(g^{\prime}, x^{\prime}, s^{\prime}\right)}
$$

we see that $\phi_{\left(g^{\prime}, x^{\prime}, s^{\prime}\right),(g, x, s)}^{f}$ is indeed a map from $A_{(g, x, s)}$ to $\left(\alpha^{f} B\right)_{\left(g^{\prime}, x^{\prime}, s^{\prime}\right)}$.

One easily checks $\phi^{f}$ is $G$-invariant. We now check $\phi^{f}$ respects the morphism support condition for morphisms in $\mathcal{O}^{G}(X ; \mathcal{A})$. By definition, the projection of $\operatorname{supp}\left(\phi^{f}\right)$ in the $X \times[1, \infty)$-direction is the same as the projection of $\operatorname{supp}(\Phi)$ in the $X \times[1, \infty)$-direction. Now by assumption, $\mathcal{E}_{\Gamma c c}^{X} \subseteq \mathcal{E}_{G c c}^{X}$. Therefore $\phi^{f}$ respects the morphism support condition in the $X \times[1, \infty)$-direction. By the morphism control condition of $\Phi$ in the $\Gamma$-direction, there is a finite set $S \subseteq \Gamma$ with the property that $\Phi_{\left(g^{\prime} f^{-1}, x^{\prime}, s^{\prime}\right),(g, x, s)} \neq 0$ implies $g^{-1} g^{\prime} f^{-1} \in S$. Therefore $\phi_{\left(g^{\prime}, x^{\prime}, s^{\prime}\right),(g, x, s)}^{f} \neq 0$ implies $g^{-1} g^{\prime} f^{-1} \in S$. This firstly shows $\phi^{f}$ respects the morphism support condition in the $G$-direction. Therefore $\phi^{f}$ is a morphism in $\mathcal{O}^{G}(X ; \mathcal{A})$. Secondly, since $S$ is finite, its image in $F$ under the natural projection $\Gamma \rightarrow F$ is finite. This implies there are only finitely many $f \in F$ with the property that there exist $g, g^{\prime} \in G$ so that $g^{-1} g^{\prime} f^{-1} \in S$. Hence there are only finitely many $f \in F$ so that $\phi^{f} \neq 0$. Therefore

$$
\phi=\sum_{f \in F} \phi^{f} \cdot f: A \rightarrow B
$$

defines a morphism in $\mathcal{O}^{G}(X ; \mathcal{A})_{\alpha}[F]$.

We now show $\tau \phi=\Phi$. For any $\left(\gamma^{\prime}, x^{\prime}, s^{\prime}\right),(\gamma, x, s) \in \Gamma \times X \times[1, \infty)$, let $\gamma^{-1} \gamma^{\prime}=g f^{-1}, g \in G, f \in$ $F$. We then have

$$
\begin{aligned}
(\tau \phi)_{\left(\gamma^{\prime}, x^{\prime}, s^{\prime}\right),(\gamma, x, s)} & =\left(\tau_{f} \phi^{f}\right)_{\left(\gamma^{\prime}, x^{\prime}, s^{\prime}\right),(\gamma, x, s)} \\
& =\left(\gamma^{-1}\right)^{*}\left[\phi_{\left(g, \gamma^{-1} x^{\prime}, s^{\prime}\right),\left(e, \gamma^{-1} x, s\right)}^{f}\right] \\
& =\left(\gamma^{-1}\right)^{*}\left[\Phi_{\left(g f^{-1}, \gamma^{-1} x^{\prime}, s^{\prime}\right),\left(e, \gamma^{-1} x, s\right)}\right] \\
& =\Phi_{\left(\gamma^{\prime}, x^{\prime}, s^{\prime}\right),(\gamma, x, s)}
\end{aligned}
$$

This shows $\tau \phi=\Phi$ and completes the proof that $\tau$ is full on morphisms.

(c) $\tau$ is faithful on morphisms: this is easy using the identity $\phi_{\left(g^{\prime}, x^{\prime}, s^{\prime}\right),(g, x, s)}^{f}:=(\tau \phi)_{\left(g^{\prime} f^{-1}, x^{\prime}, s^{\prime}\right),(g, x, s)}$. 
Therefore $\tau$ is an equivalence between $\mathcal{O}^{G}(X ; \mathcal{A})_{\alpha}[F]$ and $\mathcal{O}^{\Gamma}(X ; \mathcal{A})$ provided $\mathcal{E}_{\Gamma c c}^{X} \subseteq \mathcal{E}_{G c c}^{X}$. Now by Lemma 2.7, $\mathcal{E}_{\Gamma c c}^{X} \subseteq \mathcal{E}_{G c c}^{X}$ if $F$ is finite or the action of $\Gamma$ on $X$ is free. This completes the proof of the theorem.

Remark 3.7. One sees from the proof that the assumption $\mathcal{E}_{\Gamma c c}^{X} \subseteq \mathcal{E}_{G c c}^{X}$ is only used to show the functor $\tau$ is full. So $\tau$ is well-defined even without this assumption.

\section{Proof of the Main theorems}

In this section, we prove Theorem A and Theorem B. We firstly prove a lemma on $L$-groups which is another ingredient in proving Theorem A.

4.1. A Lemma on $L$-groups. In this subsection, we prove Lemma4.1. We will need the following well-known fact: for every group $G$ and every orientation map $w: G \longrightarrow\{ \pm 1\}$, if $W h(G)=$ $0, \tilde{K}_{0}(\mathbb{Z}[G])=0$ and $K_{i}(\mathbb{Z}[G])=0, i \leq-1$, then all the $L$-groups of $\mathbb{Z}[G]$ associated to $w$ with various decorations are naturally isomorphic (we will omit $w$ from the notations):

$$
L_{n}^{s}(\mathbb{Z}[G]) \cong L_{n}^{h}(\mathbb{Z}[G])=L_{n}^{1}(\mathbb{Z}[G]) \cong L_{n}^{0}(\mathbb{Z}[G]) \cong L_{n}^{<-1>}(\mathbb{Z}[G]) \cong \cdots \cdots L_{n}^{<-\infty>}(\mathbb{Z}[G])
$$

where $W h(G), \tilde{K}_{0}(\mathbb{Z}[G])$ and $K_{i}\left(\mathbb{Z}[G], i \leq-1\right.$ are the Whitehead group, the reduced $K_{0}$-group and the negative $K$-groups of $\mathbb{Z}[G]$ respectively. For an explanation about the decorations of various $L$-groups and a proof of the above fact, see [25, Remmark 21 on page 720 and Proposition 23 on page 721$]$.

Lemma 4.1. Let $G$ be a group. Assume $W h(G)=0, \tilde{K}_{0}(\mathbb{Z}[G])=0$ and $K_{i}(\mathbb{Z}[G])=0, i \leq-1$. Then for every group of the form $\Gamma=G \rtimes_{\alpha} \mathbb{Z}$ and every orientation map $w: \Gamma \longrightarrow\{ \pm\}$, its simple L-groups and ultimate L-groups are naturally isomorphic, i.e. $L_{n}^{s}(\mathbb{Z}[\Gamma]) \cong L_{n}^{<-\infty>}(\mathbb{Z}[\Gamma]), \forall n \in \mathbb{Z}$, naturally. If in addition $W h(G \times \mathbb{Z})=0$, then $L_{n}^{h}(\mathbb{Z}[\Gamma])$ adds to the natural isomorphisms, i.e. $L_{n}^{s}(\mathbb{Z}[\Gamma]) \cong L_{n}^{h}(\mathbb{Z}[\Gamma]) \cong L_{n}^{<-\infty>}(\mathbb{Z}[\Gamma]), \forall n \in \mathbb{Z}$, naturally.

Proof. When $\alpha: G \longrightarrow G$ is trivial, then the result follows immediately from the above well-known fact and the Shaneson splitting:

$$
\begin{gathered}
L_{n}^{<j>}(\mathbb{Z}[G \times \mathbb{Z}]) \cong L_{n}^{<j>}(\mathbb{Z}[G]) \oplus L_{n-1}^{<j-1>}(\mathbb{Z}[G]), j \leq 1 \\
L_{n}^{<s>}(\mathbb{Z}[G \times \mathbb{Z}]) \cong L_{n}^{<s>}(\mathbb{Z}[G]) \oplus L_{n-1}^{h}(\mathbb{Z}[G])
\end{gathered}
$$

which is natural with respect to the natural forgetful maps between them indexed by $s \rightarrow h \rightarrow 0 \rightarrow$ $\cdots \rightarrow-\infty$.

For the general case, we need to make use of the results of Ranicki [27]. Firstly, there is a long exact sequence [27, Page 413]:

$$
\cdots \longrightarrow L_{n}^{s}(\mathbb{Z}[G]) \longrightarrow L_{n}^{s}(\mathbb{Z}[\Gamma]) \longrightarrow L_{n-1}^{\prime}(\mathbb{Z}[G]) \longrightarrow L_{n-1}^{s}(\mathbb{Z}[G]) \longrightarrow \cdots
$$


where $L_{n-1}^{\prime}(\mathbb{Z}[G])$ is a certain intermediate $L$-group with torsion lies in $\left(1-\alpha_{*}\right)^{-1}(\bar{G})$, where

$$
1-\alpha_{*}: \tilde{K}_{1}(\mathbb{Z}[G]) \longrightarrow \tilde{K}_{1}(\mathbb{Z}[G])
$$

is the map induced by $\alpha$ and $\bar{G}$ is the image of $G$ in $\tilde{K}_{1}(\mathbb{Z}[G])$. Now by assumption, $W h(G)=0$, this implies $\bar{G}=\tilde{K}_{1}(\mathbb{Z}[G])$. Hence $\left(1-\alpha_{*}\right)^{-1}(\bar{G})=\tilde{K}_{1}(\mathbb{Z}[G])$. Therefore $L_{n}^{\prime}(\mathbb{Z}[G])=L_{n}^{h}(\mathbb{Z}[G]), \forall n \in \mathbb{Z}$ and 4.1 becomes:

$$
\cdots \longrightarrow L_{n}^{s}(\mathbb{Z}[G]) \longrightarrow L_{n}^{s}(\mathbb{Z}[\Gamma]) \longrightarrow L_{n-1}^{h}(\mathbb{Z}[G]) \longrightarrow L_{n-1}^{s}(\mathbb{Z}[G]) \longrightarrow \cdots
$$

Now there is also a well-known Wang type long exact sequence for the ultimate $L$-groups ([23, the proof of Lemma 4.2]):

$$
\cdots \longrightarrow L_{n}^{<-\infty>}(\mathbb{Z}[G]) \longrightarrow L_{n}^{<-\infty>}(\mathbb{Z}[\Gamma]) \longrightarrow L_{n-1}^{-\infty}(\mathbb{Z}[G]) \longrightarrow L_{n-1}^{<-\infty>}(\mathbb{Z}[G]) \longrightarrow \cdots
$$

The natural forgetful maps between these groups give rise to a commutative diagram of the above long exact sequences. By assumption, all these forgetful maps are isomorphisms for the $L$-groups of $\mathbb{Z}[G]$. Therefore, by the five lemma, $L_{n}^{s}(\mathbb{Z}[\Gamma]) \cong L_{n}^{<-\infty>}(\mathbb{Z}[\Gamma])$.

If in addition $W h(G \times \mathbb{Z})=0$, then by replacing $G$ by $G \times \mathbb{Z}$ in 4.1, where we use the trivial action of $\mathbb{Z}$ on $\mathbb{Z}$ (so $(G \times \mathbb{Z}) \rtimes \mathbb{Z} \cong \Gamma \times \mathbb{Z}$ ), and use the same argument as we did for $G$, we get

$$
\cdots \longrightarrow L_{n}^{s}(\mathbb{Z}[G \times \mathbb{Z}]) \longrightarrow L_{n}^{s}(\mathbb{Z}[\Gamma \times \mathbb{Z}]) \longrightarrow L_{n-1}^{h}(\mathbb{Z}[G \times \mathbb{Z}]) \longrightarrow L_{n-1}^{s}(\mathbb{Z}[G \times \mathbb{Z}]) \longrightarrow \cdots
$$

By Shaneson splitting, the above sequence naturally splits into two long exact sequences, one of which is 4.2 and another one is:

$$
\cdots \longrightarrow L_{n-1}^{h}(\mathbb{Z}[G]) \longrightarrow L_{n-1}^{h}(\mathbb{Z}[\Gamma]) \longrightarrow L_{n-2}^{0}(\mathbb{Z}[G]) \longrightarrow L_{n-2}^{0}(\mathbb{Z}[G]) \longrightarrow \cdots
$$

Now as before, 4.2, 4.3, and 4.5 together imply the natural isomorphisms $L_{n}^{s}(\mathbb{Z}[\Gamma]) \cong L_{n}^{h}(\mathbb{Z}[\Gamma]) \cong$ $L_{n}^{<-\infty>}(\mathbb{Z}[\Gamma])$.

Remark 4.2. We cannot conclude isomorphisms for the $L$-groups of $\mathbb{Z}[\Gamma]$ with other decorations. There is the issue of intermediate $L$-groups. But if we assume $W h\left(G \times \mathbb{Z}^{n}\right)=0, \forall n \in \mathbb{N}$, then all the $L$-groups of $\mathbb{Z}[\Gamma]$ are naturally isomorphic.

4.2. Proof of Theorem B. In this subsection, we prove Theorem B first. Theorem A will be a consequence of Theorem B and Lemma 4.1. We will prove the more general version of Theorem B as explained in Remark 1.5. We firstly treat the $L$-theory case. Let $\mathcal{A}$ be a right $G \rtimes_{\beta} \mathbb{Z}$ additive category with involution. View it as a $G$-additive category in the natural way. Since $G$ is torsion free, $G \rtimes_{\beta} \mathbb{Z}$ is also torsion free. This implies the $L$-theoretic assembly map $A_{L}^{\mathcal{A}}$ for $G \rtimes_{\beta} \mathbb{Z}$ in 2.2 is equivalent to the following assembly map:

$$
H_{n}^{G \rtimes_{\beta} \mathbb{Z}}\left(E\left(G \rtimes_{\beta} \mathbb{Z}\right) ; \mathbf{L}_{\mathcal{A}}^{<-\infty>}\right) \rightarrow L_{n}^{<-\infty>}\left(\mathcal{A}_{\alpha}\left[G \rtimes_{\beta} \mathbb{Z}\right]\right)
$$


where $E\left(G \rtimes_{\beta} \mathbb{Z}\right)$ is the classifying space for free $G \times_{\beta} \mathbb{Z}$-actions. See [25, Proposition 66 on page 743]. Compare [2, Theorem 8.14]. Therefore, by Theorems 2.5, 2.6, $A_{L}^{\mathcal{A}}$ for $G \times{ }_{\beta} \mathbb{Z}$ is an isomorphism if and only if

$$
L_{n}^{<-\infty>}\left(\mathcal{O}^{G \rtimes_{\beta} \mathbb{Z}}\left(E\left(G \rtimes_{\beta} \mathbb{Z}\right), \mathcal{A}\right)\right)=0, \forall n \in \mathbb{Z}
$$

Now by Theorem 3.6, we have an equivalence of additive categories with involutions

$$
\mathcal{O}^{G \rtimes_{\beta} \mathbb{Z}}\left(E\left(G \rtimes_{\beta} \mathbb{Z}\right), \mathcal{A}\right) \cong \mathcal{O}^{G}\left(E\left(G \rtimes_{\beta} \mathbb{Z}\right), \mathcal{A}\right)_{\beta}[\mathbb{Z}]
$$

since the action of $G \rtimes_{\beta} \mathbb{Z}$ on $E\left(G \rtimes_{\beta} \mathbb{Z}\right)$ is free. Denote $\mathcal{O}^{G}\left(E\left(G \rtimes_{\beta} \mathbb{Z}\right), \mathcal{A}\right)$ by $\tilde{\mathcal{A}}$. Note that by the characterization properties of classifying spaces, $E\left(G \rtimes_{\beta} \mathbb{Z}\right)$ is also a model for the classifying space for free $G$-actions. Now by assumption, the $L$-theoretic FJC holds for $G$ with coefficient in $\mathcal{A}$, therefore, by Theorems 2.5, 2.6, we have

$$
L_{n}^{<-\infty>}(\tilde{\mathcal{A}})=0, \forall n \in \mathbb{Z}
$$

Now by applying the Wang type long exact sequence 4.3 to $L_{*}^{<-\infty>}\left(\tilde{\mathcal{A}}_{\beta}[\mathbb{Z}]\right)$, which is well-known when the coefficient is a ring and can be extended to with twisted coefficient in any additive category (see [2, proof of Theorem 8.14]), we immediately get that

$$
L_{n}^{<-\infty>}\left(\tilde{\mathcal{A}}_{\beta}[\mathbb{Z}]\right)=0, \forall n \in \mathbb{Z}
$$

This implies

$$
L_{n}^{<-\infty>}\left(\mathcal{O}^{G \rtimes_{\beta} \mathbb{Z}}\left(E\left(G \rtimes_{\beta} \mathbb{Z}\right), \mathcal{A}\right)\right)=0, \forall n \in \mathbb{Z}
$$

Therefore the $L$-theoretic FJC with coefficient in $\mathcal{A}$ holds for $G \rtimes_{\beta} \mathbb{Z}$. This proves part (1) of Theorem B.

We now turn into part (2) of Theorem B. When $R$ is regular and $G \rtimes_{\beta} \mathbb{Z}$ is torsion free, the $K$-theoretic assembly map $A_{K}^{R}$ for $G \rtimes_{\beta} \mathbb{Z}$ in 2.1 is equivalent to the following assembly map:

$$
H_{n}^{G \rtimes_{\beta} \mathbb{Z}}\left(E\left(G \rtimes_{\beta} \mathbb{Z}\right) ; \mathbf{K}_{\mathcal{A}}\right) \rightarrow K_{n}\left(\mathcal{A}_{\alpha}\left[G \rtimes_{\beta} \mathbb{Z}\right]\right)
$$

Similar to the argument as in the $L$-theoretic case, we see that the above assembly map is an isomorphism if and only if

$$
K_{n}\left(\mathcal{O}^{G}\left(E\left(G \rtimes_{\beta} \mathbb{Z}\right), R\right)_{\beta}[\mathbb{Z}]\right)=0, \forall n \in \mathbb{Z}
$$

Now, there is a Wang type long exact sequence for the non-connective $K$-theory of $\mathcal{O}^{G}\left(E\left(G \rtimes_{\beta}\right.\right.$ $\mathbb{Z}), R)_{\beta}[\mathbb{Z}$. This is well-known when the coefficient is a ring and has been recently generalized by Lück-Steimle [26, Theorem 0.1, Remark 0.2] to with coefficient in any additive category. Using this sequence and the assumption that the $K$-theoretic FJC holds for $G$ with coefficient in $R$, we see that

$$
K_{n}\left(\mathcal{O}^{G}\left(E\left(G \rtimes_{\beta} \mathbb{Z}\right), R\right)_{\beta}[\mathbb{Z}]\right) \cong N K_{n}\left(\mathcal{O}^{G}\left(E\left(G \rtimes_{\beta} \mathbb{Z}\right), R\right) ; \beta\right) \oplus N K_{n}\left(\mathcal{O}^{G}\left(E\left(G \rtimes_{\beta} \mathbb{Z}\right), R\right) ; \beta\right)
$$


Denote the Nil-groups $N K_{n}\left(\mathcal{O}^{G}\left(E\left(G \rtimes_{\beta} \mathbb{Z}\right), R\right) ; \beta\right)$ as defined in [26] by $N i l_{n, R}^{G \rtimes_{\beta} \mathbb{Z}}$, then

$$
K_{n}\left(\mathcal{O}^{G}\left(E\left(G \rtimes_{\beta} \mathbb{Z}\right), R\right)_{\beta}[\mathbb{Z}]\right)=0
$$

if and only if $N i l_{n, R}^{G \rtimes_{\beta} \mathbb{Z}}=0$. This proves part (2) of Theorem B.

4.3. Proof of Theorem A. We now prove Theorem A. Let us assume $G \in \mathcal{F} \mathcal{J}$. If $G$ is not torsion free, then there is nothing to prove, since it cannot be realized as the fundamental group of a closed aspherical manifold. So let us assume $G$ is torsion free. Since $G$ satisfies the $K$-theoretic FJC with coefficient in $\mathbb{Z}$, it follows that

$$
W h(G)=0, \quad \tilde{K}_{0}(\mathbb{Z}[G])=0, \quad K_{i}(\mathbb{Z}[G])=0, i \leq-1
$$

See [25, Conjecture 1 on page 708, Conjecture 3 on page 710]. Therefore Lemma 4.1 applies and we have, for every orientation map $w: G \rtimes \mathbb{Z} \longrightarrow\{ \pm\}$, natural isomorphisms

$$
L_{n}^{s}(\mathbb{Z}[G \rtimes \mathbb{Z}]) \cong L_{n}^{<-\infty>}(\mathbb{Z}[G \rtimes \mathbb{Z}]), \forall n \in \mathbb{Z}
$$

Because $G$ is torsion free and satisfies the $L$-theoretic FJC with coefficient in $\mathbb{Z}$, Theorem B applies and the $L$-theoretic FJC with coefficient in $\mathbb{Z}$ holds for $G \rtimes \mathbb{Z}$. Therefore, by a standard surgery long exact sequence argument, we see that the simple Borel conjecture holds for $G \rtimes \mathbb{Z}$. For the convenience of the reader, let us sketch the main idea, more details can be found in [25, Theorem 28 on page 723], [17, pages 26-28] . Let $M$ be a closed aspherical manifold of dimension $n \geq 5$ with $\pi_{1}(M) \cong G \rtimes \mathbb{Z}$. Let $S^{S}(M)$ be its simple topological structure set (an abelian group indeed). It consists of the equivalence classes of all simple homotopy equivalence $f: N \longrightarrow M$, from another closed manifold $N$ to $M$. Two such maps $f: N \longrightarrow M, f^{\prime}: N^{\prime} \longrightarrow M$ are equivalent if there is a homeomorphism $g: N \longrightarrow N^{\prime}$ so that $f^{\prime} \circ g$ is homotopic to $f$. Therefore the simple Borel conjecture holds for $G$ if and only if $S^{s}(M)$ consists of one point. Now there is a surgery long exact sequence for $S^{s}(M)$ :

$$
\cdots \longrightarrow \mathcal{N}_{n+1}(M) \stackrel{\sigma_{n+1}}{\longrightarrow} L_{n+1}^{s}(\mathbb{Z}[G \rtimes \mathbb{Z}]) \stackrel{\partial_{n+1}}{\longrightarrow} S^{s}(M) \stackrel{\eta_{n}}{\longrightarrow} \mathcal{N}_{n}(M) \stackrel{\sigma_{n}}{\longrightarrow} L_{n}^{s}(\mathbb{Z}[G \rtimes \mathbb{Z}])
$$

For each $i \geq n$, the assembly map 2.2 and the above sequence fit into the following commutative diagram

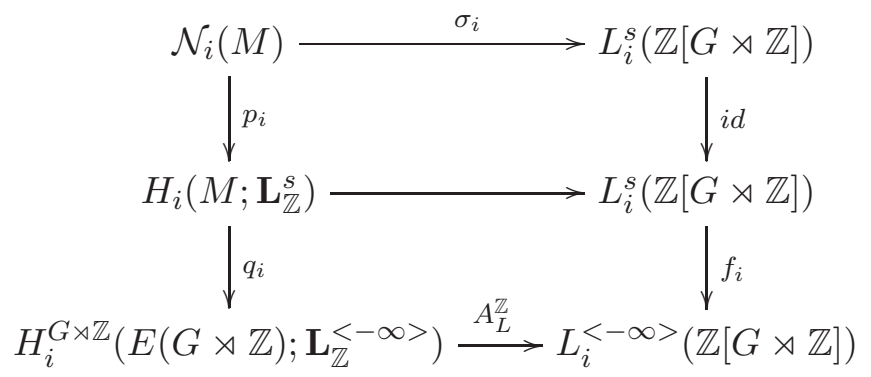


We have showed that $f_{i}$ and $A_{L}^{\mathbb{Z}}$ are isomorphisms for all $i \geq n . q_{i}$ is also an isomorphism since

$$
H_{i}^{G \rtimes \mathbb{Z}}\left(E(G \rtimes \mathbb{Z}) ; \mathbf{L}_{\mathbb{Z}}^{<-\infty>}\right) \cong H_{i}\left(M ; \mathbf{L}_{\mathbb{Z}}^{<-\infty>}\right) \cong H_{i}\left(M ; \mathbf{L}_{\mathbb{Z}}^{s}\right)
$$

where the first isomorphism comes from, by viewing $M=B(G \rtimes \mathbb{Z})=E(G \rtimes \mathbb{Z}) / G \rtimes \mathbb{Z}$, the induction structure of an equivariant homology, see [25, Remark 61 on page 736], and the second isomorphism is because the two homology theories $H_{*}\left(-; \mathbf{L}_{\mathbb{Z}}^{s}\right), H_{*}\left(-; \mathbf{L}_{\mathbb{Z}}^{<-\infty>}\right)$ are naturally isomorphic (since

they are naturally isomorphic on a point). Now it is a fact from surgery theory that $p_{i}$ is injective when $i=n$, and bijective when $i>n$. This implies that $\sigma_{n}$ is injective and $\sigma_{n+1}$ is bijective. Therefore $S^{s}(M)$ is trivial and the simple Borel conjecture holds for $G \rtimes \mathbb{Z}$.

If in addition $W h(G \times \mathbb{Z})=0$, then by Lemma 4.1, we have

$$
L_{n}^{h}(\mathbb{Z}[G \rtimes \mathbb{Z}]) \cong L_{n}^{<-\infty>}(\mathbb{Z}[G \rtimes \mathbb{Z}]), \forall n \in \mathbb{Z}
$$

This implies, by using the surgery long exact sequence for the structure set $S^{h}(M)$, which consists of $h$-cobordant equivalence classes of all homotopy equivalent maps to $M$, and a similar argument as above, that $S^{h}(M)$ consists of a single element. This implies the bordism Borel conjecture holds for $G \rtimes \mathbb{Z}$. This completes the proof of Theorem A.

\section{REFERENCES}

[1] Douglas R. Anderson, Francis X. Connolly, Steven C. Ferry, and Erik K. Pedersen. Algebraic K-theory with continuous control at infinity. J. Pure Appl. Algebra, 94(1):25-47, 1994.

[2] A. Bartels, F. T. Farrell, and W. Lück. The Farrell-Jones conjecture for cocompact lattices in virtually connected Lie groups. J. Amer. Math. Soc., 27(2):339-388, 2014.

[3] Arthur Bartels, Siegfried Echterhoff, and Wolfgang Lück. Inheritance of isomorphism conjectures under colimits. In K-theory and noncommutative geometry, EMS Ser. Congr. Rep., pages 41-70. Eur. Math. Soc., Zürich, 2008.

[4] Arthur Bartels, Tom Farrell, Lowell Jones, and Holger Reich. On the isomorphism conjecture in algebraic $K$ theory. Topology, 43(1):157-213, 2004.

[5] Arthur Bartels and Wolfgang Lück. The Borel conjecture for hyperbolic and CAT(0)-groups. Ann. of Math. (2), 175(2):631-689, 2012.

[6] Arthur Bartels, Wolfgang Lück, and Holger Reich. The K-theoretic Farrell-Jones conjecture for hyperbolic groups. Invent. Math., 172(1):29-70, 2008.

[7] Arthur Bartels, Wolfgang Lück, Holger Reich, and Henrik Rüping. K- and L-theory of group $\operatorname{rings}$ over $G L_{n}(\mathbf{Z})$. Publ. Math. Inst. Hautes Études Sci., 119:97-125, 2014.

[8] Arthur Bartels and Holger Reich. Coefficients for the Farrell-Jones conjecture. Adv. Math., 209(1):337-362, 2007.

[9] M. Cárdenas and E. K. Pedersen. On the Karoubi filtration of a category. K-Theory, 12(2):165-191, 1997.

[10] T. A. Chapman. Topological invariance of Whitehead torsion. Amer. J. Math., 96:488-497, 1974.

[11] James F. Davis and Wolfgang Lück. Spaces over a category and assembly maps in isomorphism conjectures in $K$ - and $L$-theory. K-Theory, 15(3):201-252, 1998.

[12] Michael W. Davis. Poincaré duality groups. In Surveys on surgery theory, Vol. 1, volume 145 of Ann. of Math. Stud., pages 167-193. Princeton Univ. Press, Princeton, NJ, 2000.

[13] F. T. Farrell and L. E. Jones. Isomorphism conjectures in algebraic K-theory. J. Amer. Math. Soc., 6(2):249-297, 1993. 
[14] F. Thomas Farrell and Xiaolei Wu. The Farrell-Jones conjecture for some nearly crystallographic groups. Algebr. Geom. Topol., 15(3):1667-1690, 2015.

[15] F. Thomas Farrell and Xiaolei Wu. Isomorphism conjecture for Baumslag-Solitar groups. Proc. Amer. Math. Soc., 143(8):3401-3406, 2015.

[16] Tom Farrell and Xiaolei Wu. The Farrell-Jones conjecture for the solvable Baumslag-Solitar groups. Math. Ann., 359(3-4):839-862, 2014.

[17] Steven C. Ferry, Andrew Ranicki, and Jonathan Rosenberg. A history and survey of the Novikov conjecture. In Novikov conjectures, index theorems and rigidity, Vol. 1 (Oberwolfach, 1993), volume 226 of London Math. Soc. Lecture Note Ser., pages 7-66. Cambridge Univ. Press, Cambridge, 1995.

[18] Giovanni Gandini, Sebastian Meinert, and Henrik Rüping. The Farrell-Jones conjecture for fundamental groups of graphs of abelian groups. Groups, Geometry, and Dynamics, 9(3):783-792, 2015.

[19] Ian Hambleton, Erik K. Pedersen, and David Rosenthal. Assembly maps for group extensions in $K$-theory and L-theory with twisted coefficients. Pure Appl. Math. Q., 8(1):175-197, 2012.

[20] N. Higson, V. Lafforgue, and G. Skandalis. Counterexamples to the Baum-Connes conjecture. Geom. Funct. Anal., 12(2):330-354, 2002.

[21] Nigel Higson, Erik Kjær Pedersen, and John Roe. $C^{*}$-algebras and controlled topology. K-Theory, 11(3):209-239, 1997.

[22] Holger Kammeyer, Wolfgang Lück, and Henrik Rüping. The Farrell-Jones conjecture for arbitrary lattices in virtually connected Lie groups. to appear in Geometry and Topology, arXiv:1401.0876.

[23] Wolfgang Lück. $K$ - and $L$-theory of the semi-direct product of the discrete 3-dimensional Heisenberg group by $\mathbb{Z} / 4$. Geom. Topol., 9:1639-1676 (electronic), 2005.

[24] Wolfgang Lück. Survey on aspherical manifolds. European Congress of Mathematics, pages 53-82, 2010.

[25] Wolfgang Lück and Holger Reich. The Baum-Connes and the Farrell-Jones conjectures in $K$ - and $L$-theory. Handbook of K-theory. Vol. 1, 2, pages 703-842, 2005.

[26] Wolfgang Lück and Wolfgang Steimle. A twisted Bass-Heller-Swan decomposition for the non-connective $K$ theory of additive categories. to appear in Forum Mathematicum, arXiv: 1309.1353.

[27] A. A. Ranicki. Algebraic L-theory. III. Twisted Laurent extensions. pages 412-463. Lecture Notes in Mathematics, Vol. 343. Springer, Berlin, Proc. Conf. Seattle Res. Center, Battelle Memorial Inst., 1972.

[28] Henrik Rüping. The Farrell-Jones conjecture for S-arithmetic groups. arXiv:1309.7236.

[29] C. T. C. Wall. List of problems. volume 36 of London Math. Soc. Lecture Note Ser., pages 369-394. Cambridge Univ. Press, Cambridge-New York, Proc. Sympos., Durham, 1977.

[30] Christian Wegner. The Farrell-Jones conjecture for virtually solvable groups. to appear in Journal of Topology, arXiv:1308.2432.

[31] Christian Wegner. The K-theoretic Farrell-Jones conjecture for CAT(0)-groups. Proc. Amer. Math. Soc., 140(3):779-793, 2012.

[32] Guoliang Yu. The coarse Baum-Connes conjecture for spaces which admit a uniform embedding into Hilbert space. Invent. Math., 139(1):201-240, 2000.

Department of Mathematics, Vanderbilt University, 1326 Stevenson Center, Nashville, TN 37240

E-mail address: kun.wang@vanderbilt.edu 